\title{
Shape and Polarity
}

outline circular - 156

outline elliptic - 158

outline lobate - 160

outline triangular - 163

outline quadrangular - 166

outline polygonal - 167

P/E-ratio, oblate - 168

$\mathrm{P} / \mathrm{E}$-ratio, isodiametric - 171

P/E-ratio, prolate - 173

isopolar -176

heteropolar - 178

shape - 181

saccus/saccate, corpus - 188

saccus, monosaccate - 189

saccus, bisaccate - 190

saccus, trisaccate -193

infoldings, apertures sunken - 194

infoldings, boat-shaped - 196

infoldings, cup-shaped - 199

infoldings, interapertural area sunken - 201

infoldings, irregular -203 
outline circular

outline describes the contour of pollen grains in polar and/or equatorial view
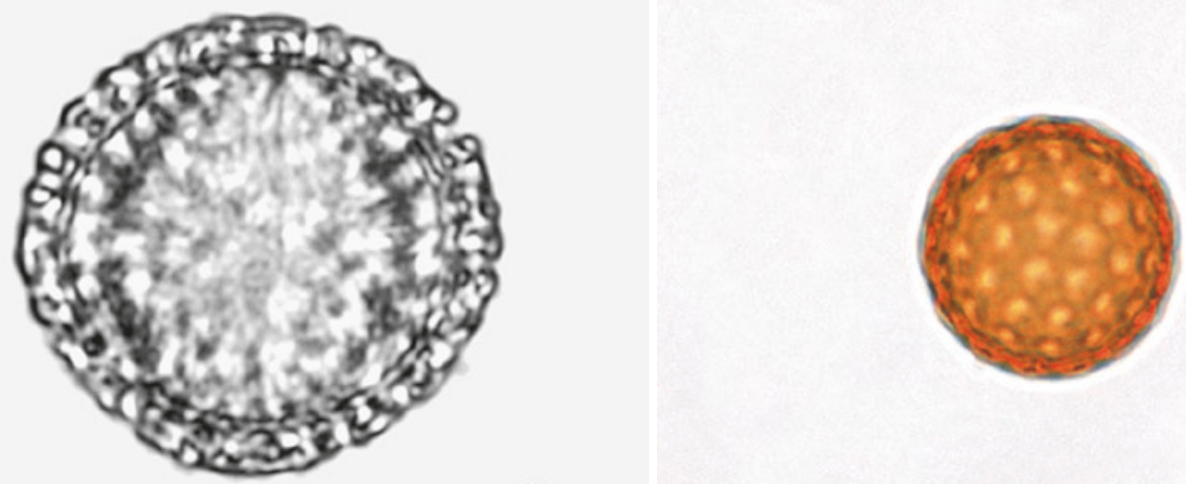

$10 \mu \mathrm{m}$
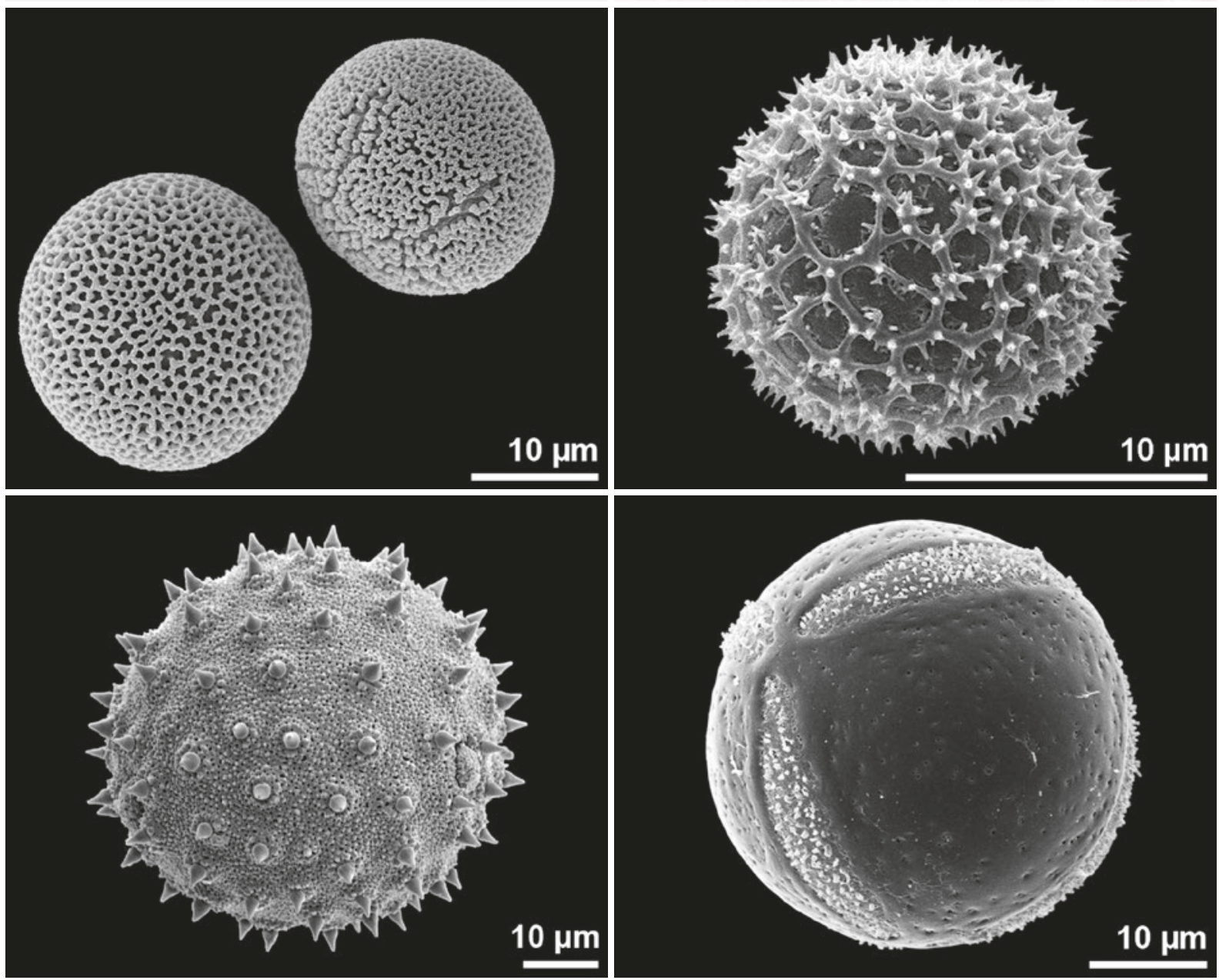

- Ligustrum sp., Oleaceae

Chenopodiaceae

fossil, middle Miocene, Austria, equatorial view

Anthurium ovatifolium, Araceae
porate

- Hedyosmum scaberrimum, Chloranthaceae

polychotomosulcate

Abutilon theophrasti, Malvaceae

triporate, equatorial view

Corydalis ophiocarpa, Papaveraceae

hexacolpate 

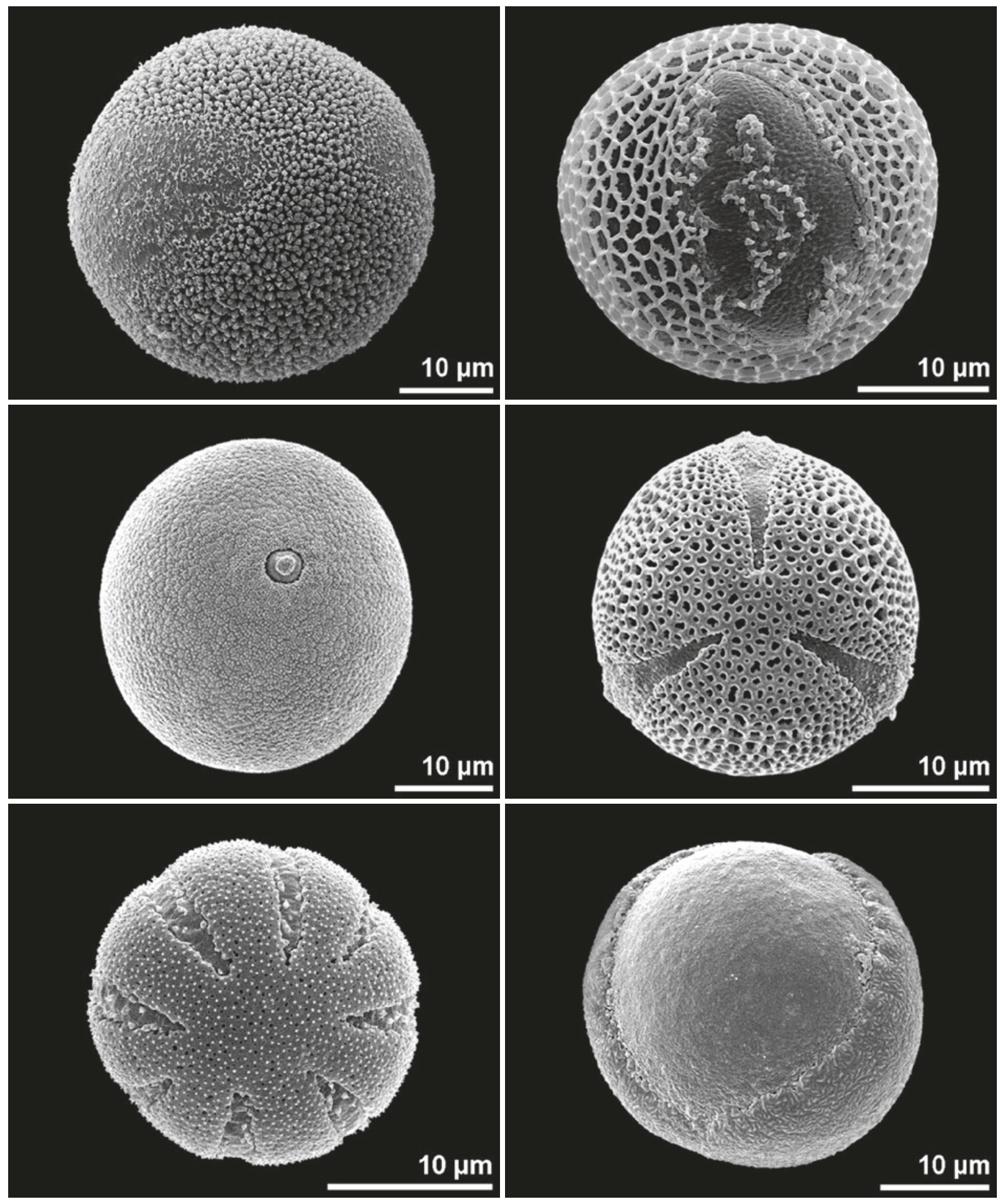

- Mayna odorata, Achariaceae

- Saruma henryi, Aristolochiaceae dicolpate, polar view sulcate, distal polar view

Fraxinus ornus, Oleaceae

Phleum pratense, Poaceae ulcerate, distal polar view

tricolporate, polar view 
outline elliptic
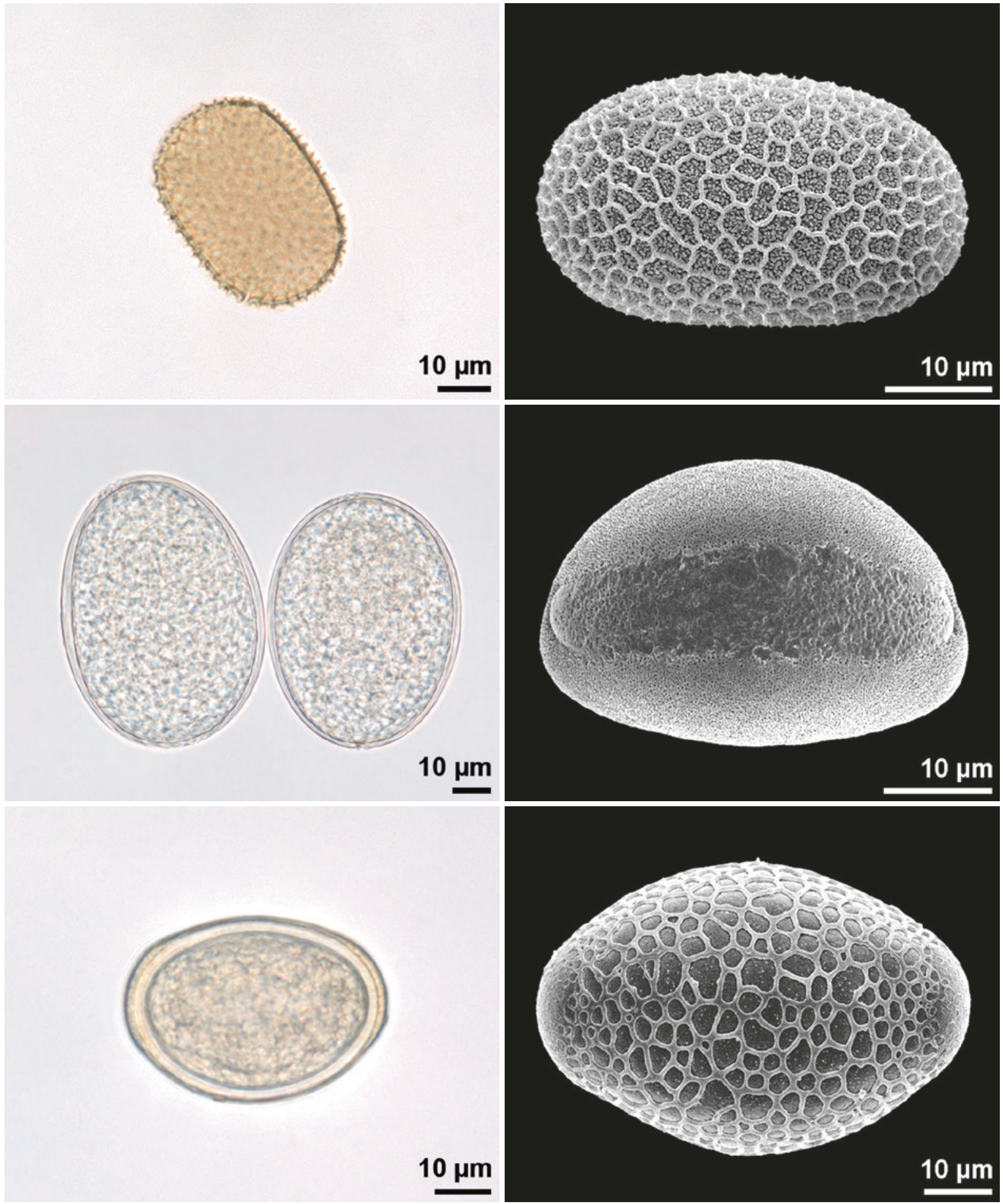

- Impatiens parviflora, Balsaminaceae

Impatiens parviflora, Balsaminaceae tetracolpate, polar view

- Allium oleraceum, Amaryllidaceae sulcate, distal polar view

Amorphophallus interruptus, Araceae inaperturate, hydrated pollen

Aechmea dealbata, Bromeliaceae 

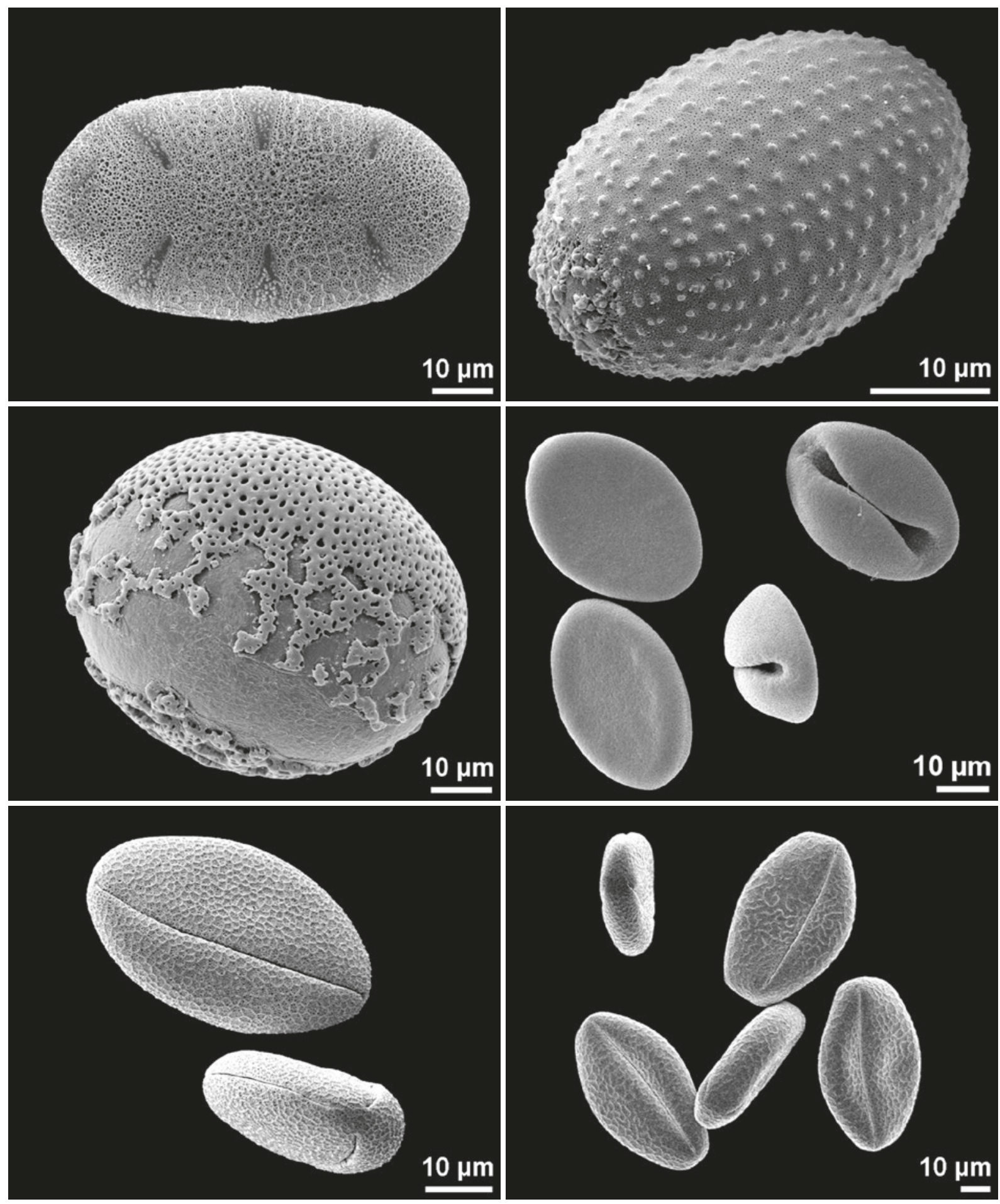

Salvia coccinea, Lamiaceae

hexacolpate, polar view

- Commelina erecta, Commelinaceae

sulcate, proximal polar view

Billbergia porteana, Bromeliaceae sulcate, distal polar view

Zamia loddigesii, Zamiaceae sulcate, dry pollen

Physostegia virginiana, Lamiaceae

Galeopsis tetrahit, Lamiaceae

- tricolpate, dry pollen

tricolpate, dry pollen 


\section{outline lobate}

outline in polar view of a pollen grain with bulged interapertural areas (mainly in dry pollen grains)
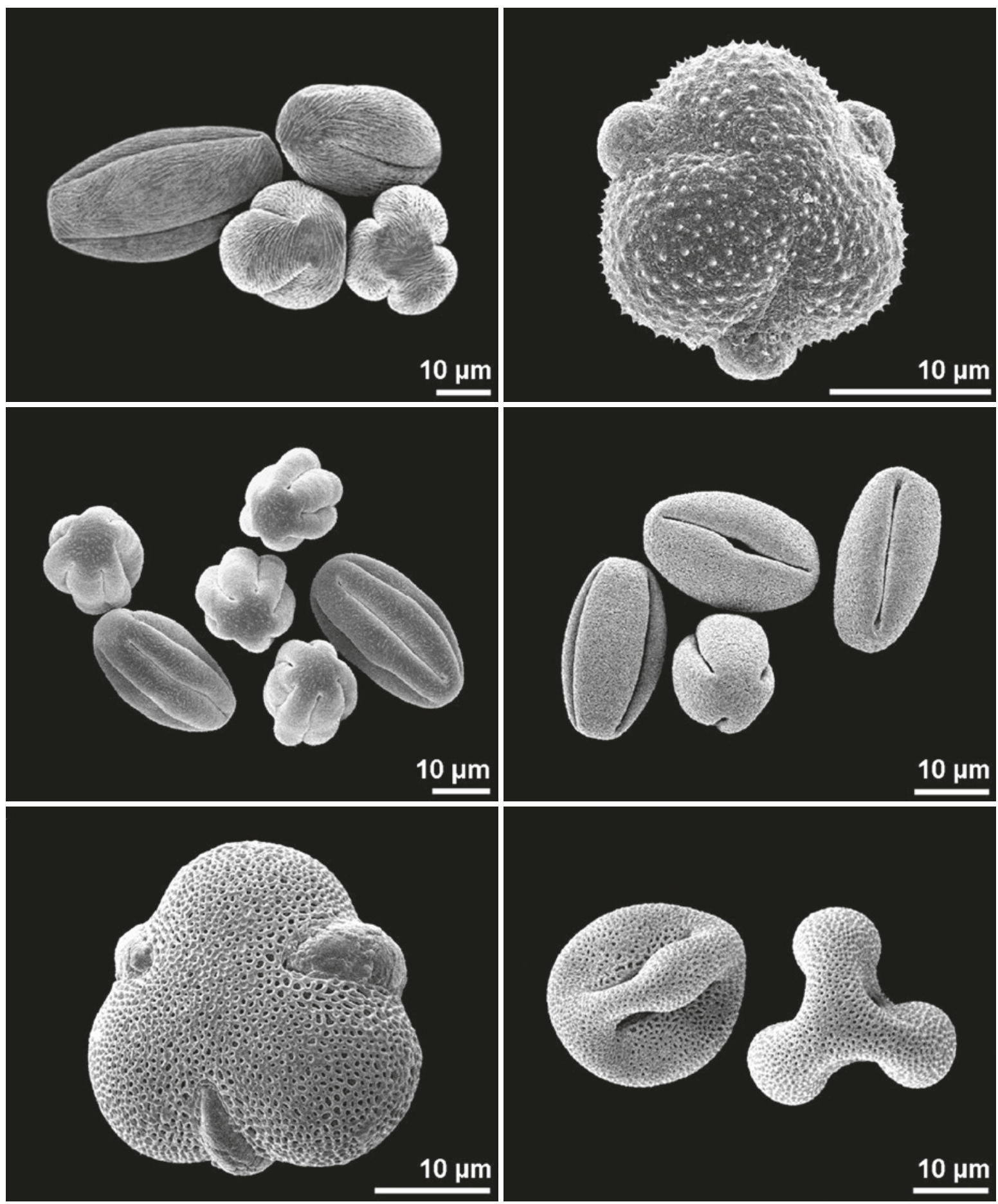

\footnotetext{
- Acer pseudoplatanus, Sapindaceae tricolpate, dry pollen

Sanguisorba officinalis, Rosaceae hexacolporate, dry pollen

\section{Gunnera tinctoria, Gunneraceae} - tricolpate, polar view
}

- Artemisia pontica, Asteraceae tricolporate, polar view

- Orthilia secunda, Ericaceae tricolporate, dry pollen 

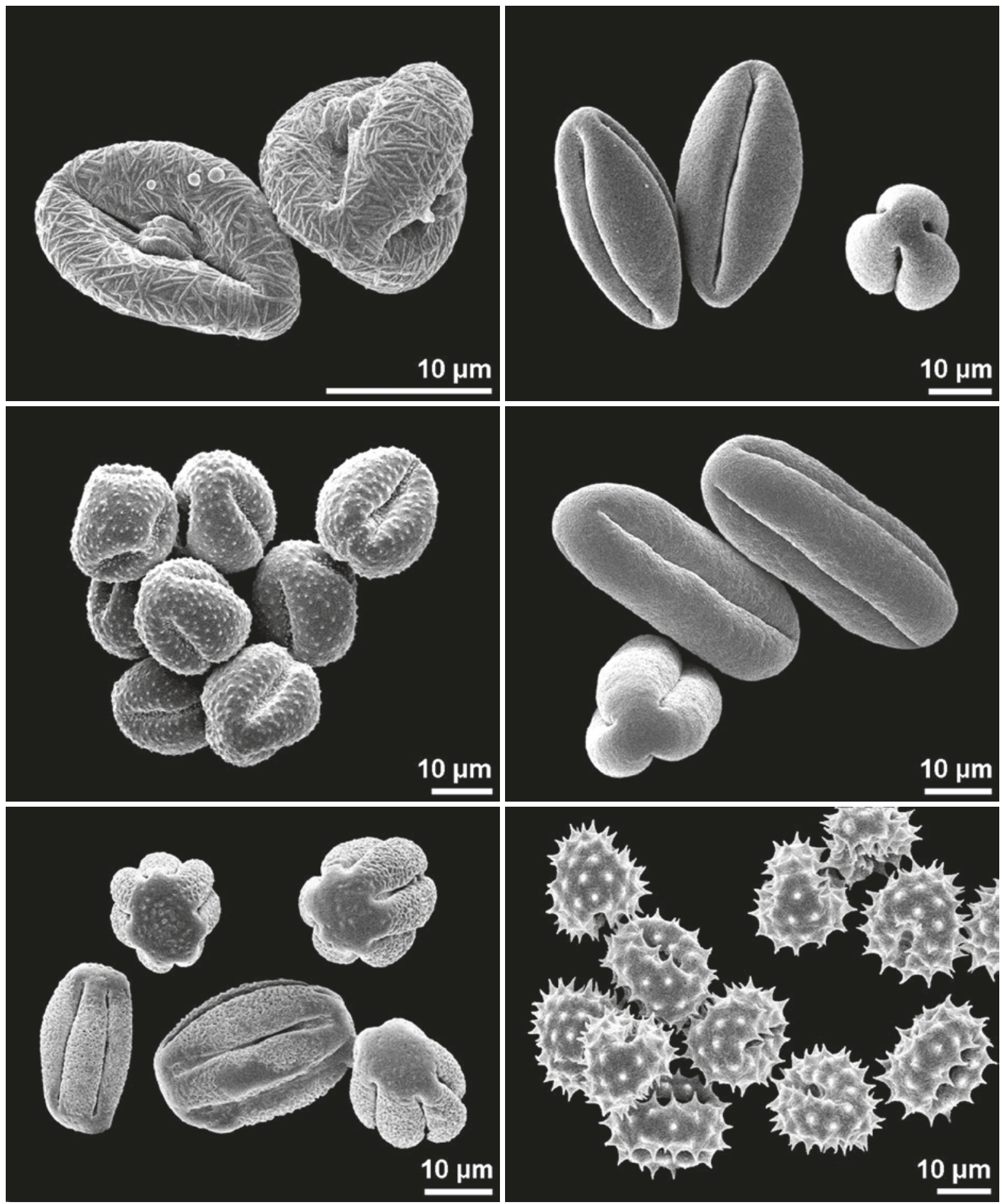

- Sedum rupestre, Crassulaceae

- Viola alba, Violaceae tricolporate, dry pollen

tricolporate, dry pollen

- Clematis heracleifolia, Ranunculaceae tricolpate, dry pollen

- Sanicula europaea, Apiaceae

tricolporate, dry pollen

Bellis perennis, Asteraceae

Pinguicula ehlersiae, Lentibulariaceae

- stephanocolporate, dry pollen

tricolporate, dry pollen 

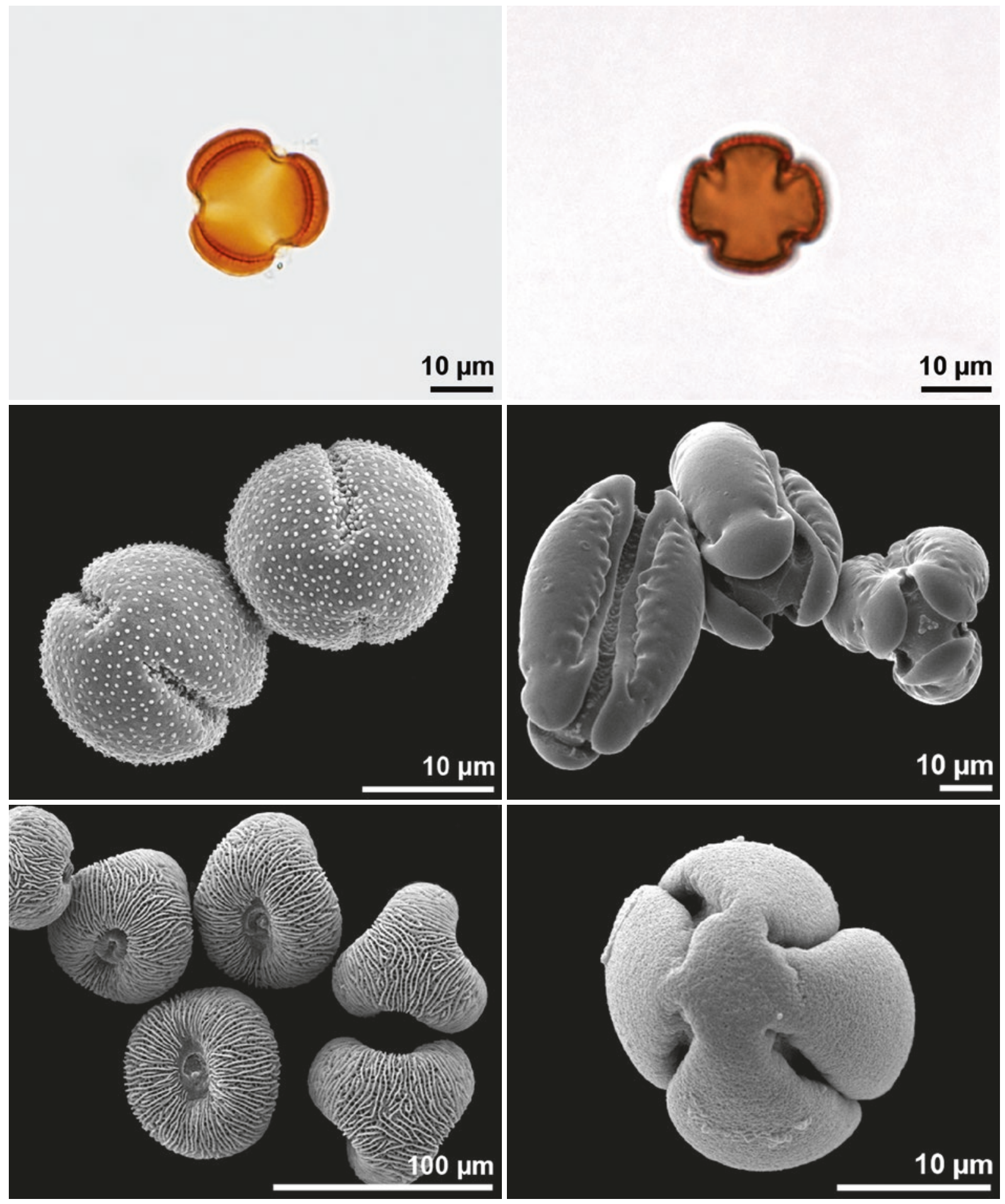

- Artemisia sp., Asteraceae

- Nicotiana tabacum, Solanaceae tricolporate, polar view tetracolporate, polar view Hypecoum imberbe, Papaveraceae
dicolpate, polar view

Barringtonia asiatica, Lecythidaceae tricolpate, dry pollen

Pelargonium punctatum, Geraniaceae - tricolporate, dry pollen

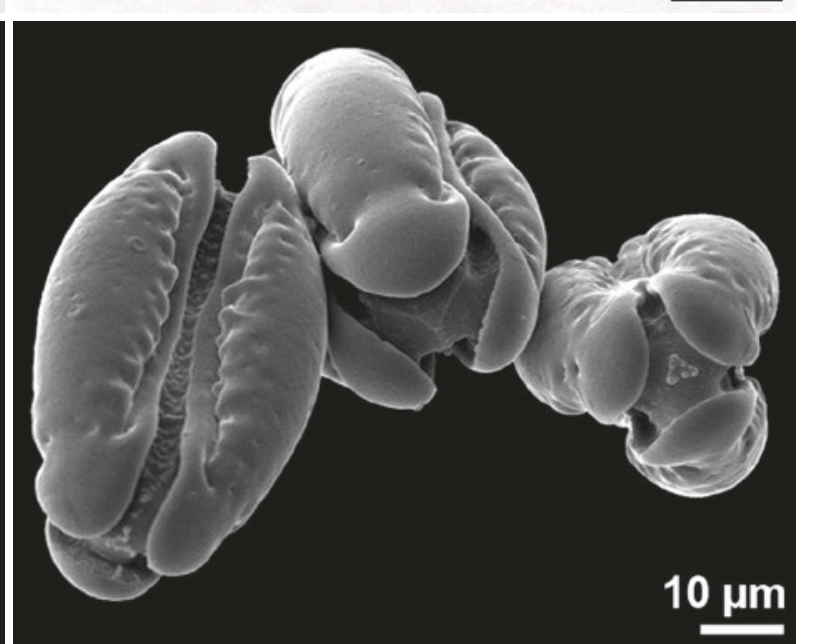




\section{outline triangular}

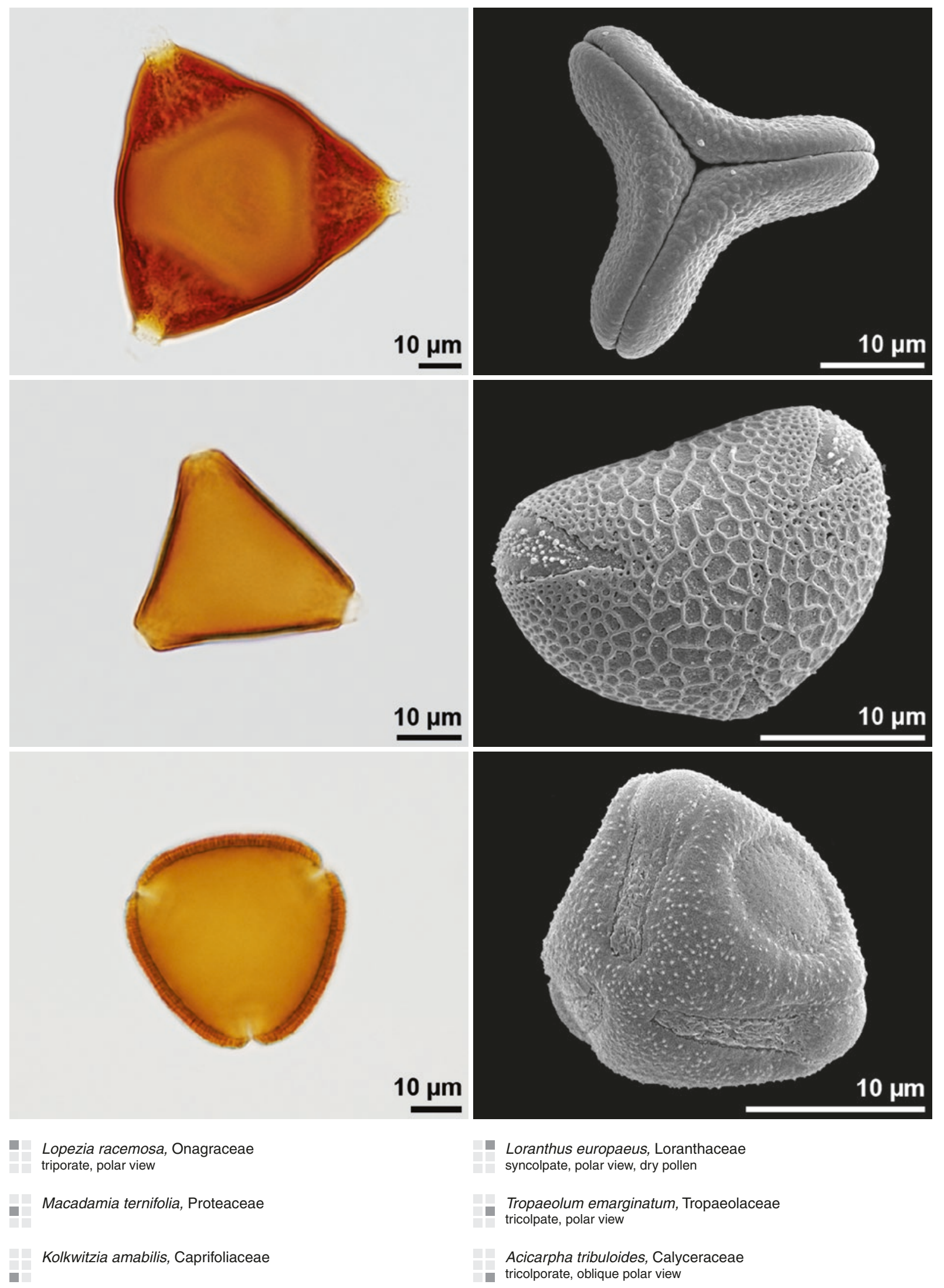



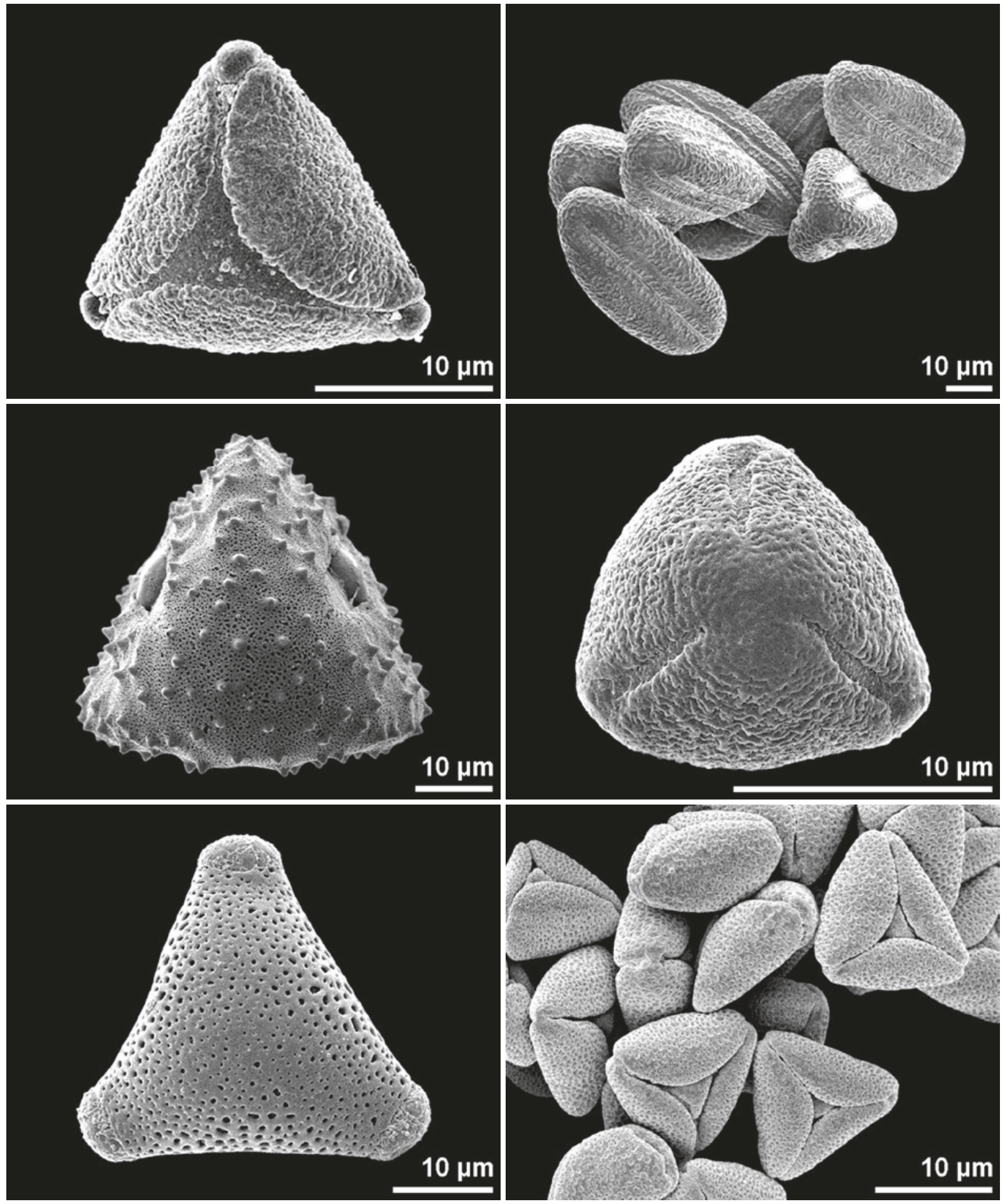

- Callistemon coccineus, Myrtaceae tricolporate, synaperturate, polar view

Echinops ritro, Asteraceae

tricolporate, polar view

Paullinia tomentosa, Sapindaceae

triporate, polar view
Hypoestes phyllostachya, Acanthaceae tricolporate, dry pollen

Bupleurum rotundifolium, Apiaceae tricolporate, polar view

Primula denticulata, Primulaceae tricolporate, synaperturate, dry pollen 

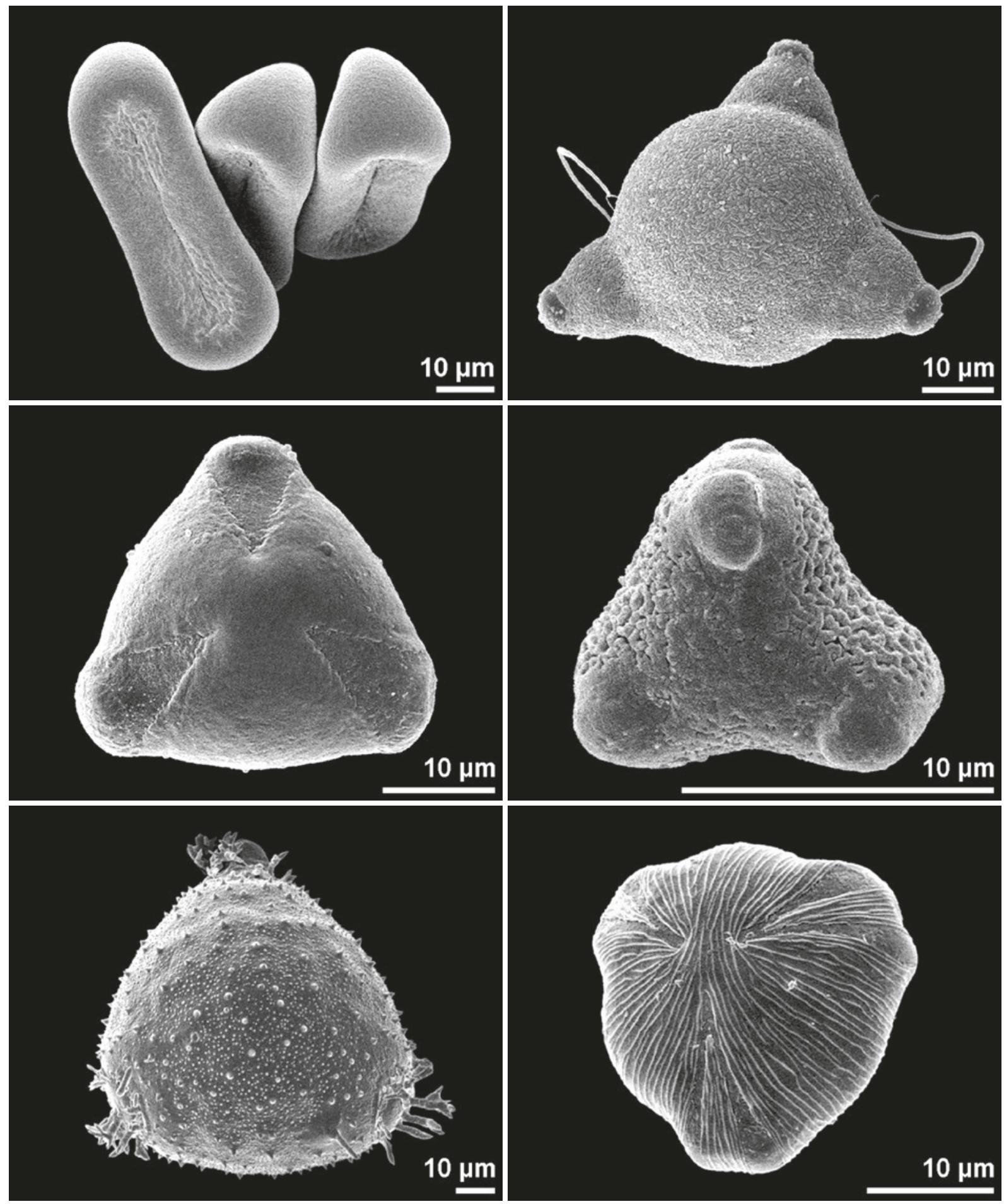

- Orlaya grandiflora, Apiaceae

- Circaea lutetiana, Onagraceae tricolporate, dry pollen

triporate, pollen with viscin threads, polar view

Sempervivum globiferum, Crassulaceae

- Cunonia capensis, Cunoniaceae

tricolporate, polar view

hexaporate, polar view

Potentilla inclinata, Rosaceae

Dipsacus fullonum, Caprifoliaceae

n triporate, polar view

- tricolporate, polar view 
outline quadrangular
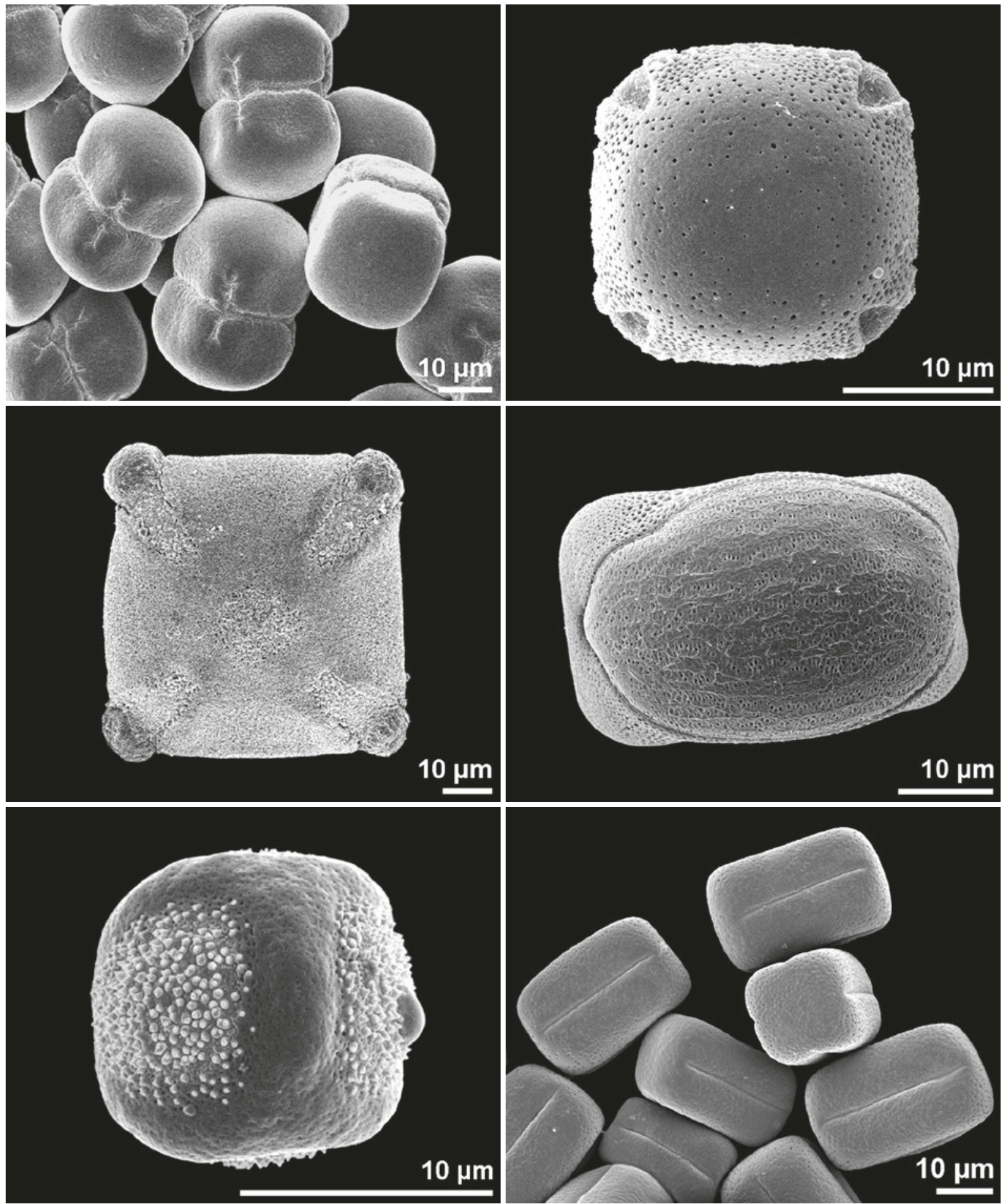

- Anchusa officinalis, Boraginaceae

- Nonea pulla, Boraginaceae tetracolporate, dry pollen tetracolporate, polar view

Viola tricolor, Violaceae
tetracolporate, polar view

- Eremurus robustus, Xanthorrhoeaceae sulcate, distal polar view 


\section{outline polygonal}
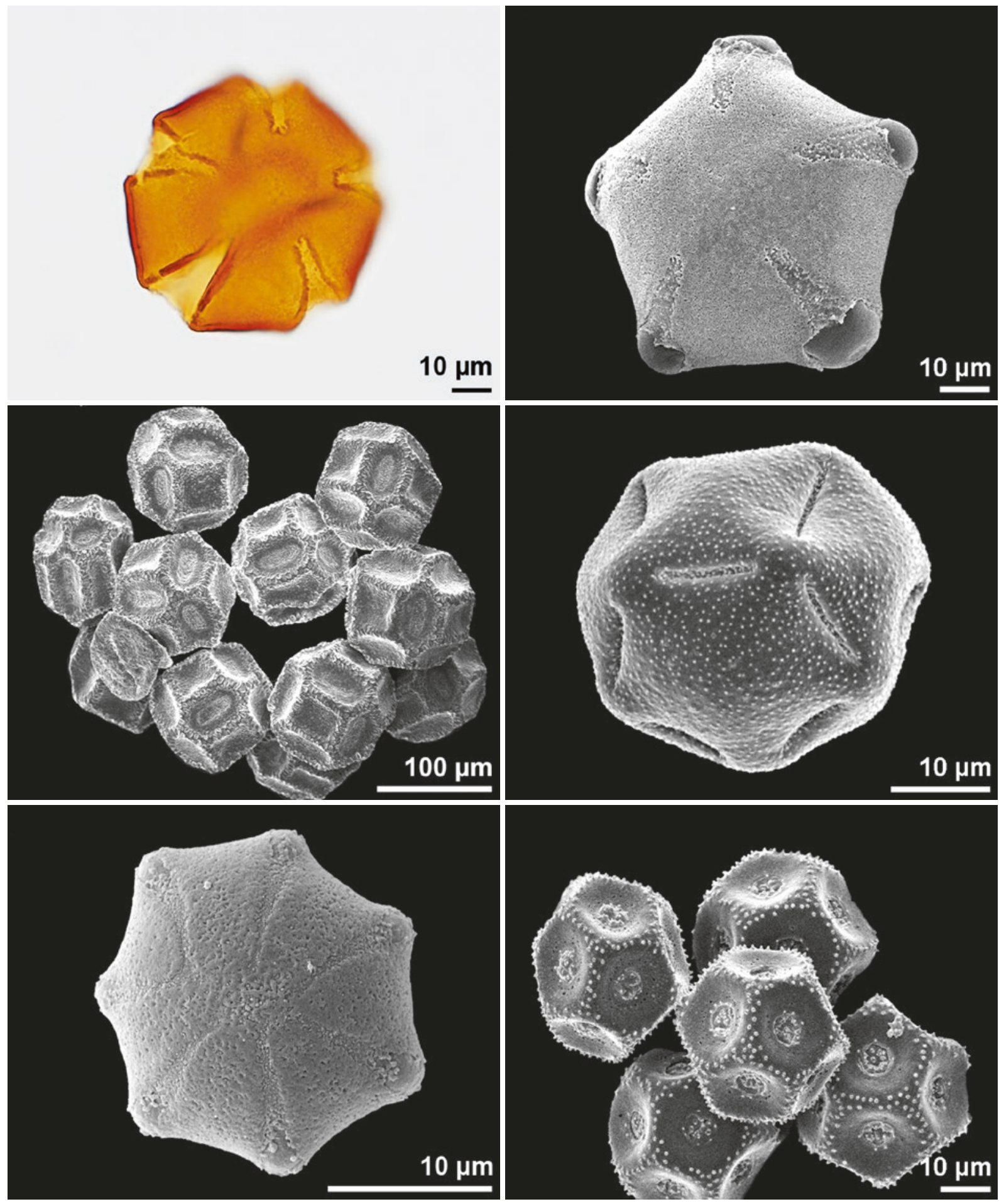

- Viola arvensis, Violaceae

- Viola arvensis, Violaceae pentacolporate, polar view pentacolporate, polar view

- Opuntia basilaris, Cactaceae

Talinum paniculatum, Talinaceae
pantocolpate, dry pollen

Sarracenia alata, Sarraceniaceae

- stephanocolporate, polar view

Stellaria holostea, Caryophyllaceae

- pantoporate, dry pollen 


\section{P/E-ratio, oblate}

P/E-ratio refers to the length of the polar axis between the two poles compared to the equatorial diameter oblate: pollen grain with a polar axis shorter than the equatorial diameter

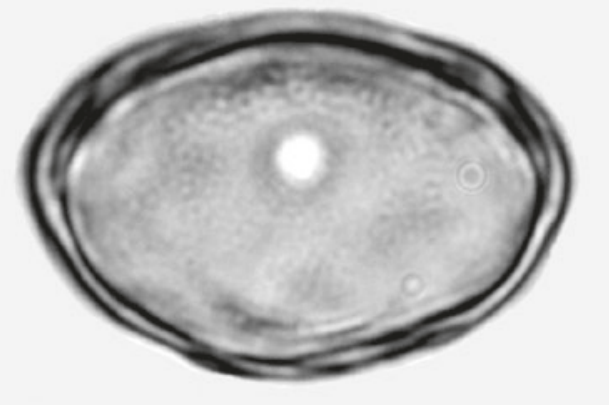

$10 \mu \mathrm{m}$
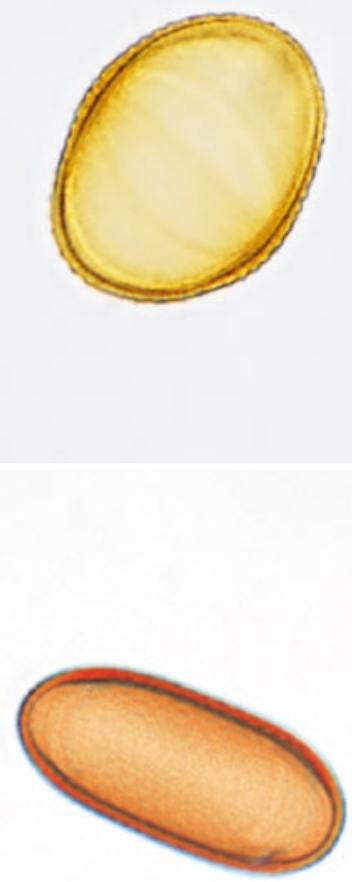

- Carya sp., Juglandaceae fossil, middle Miocene, Austria, triporate, equatorial view

- Plectranthus esculentus, Lamiaceae hexacolpate, equatorial view

Impatiens sp., Balsaminaceae

- tetracolpate, equatorial view

$10 \mu \mathrm{m}$
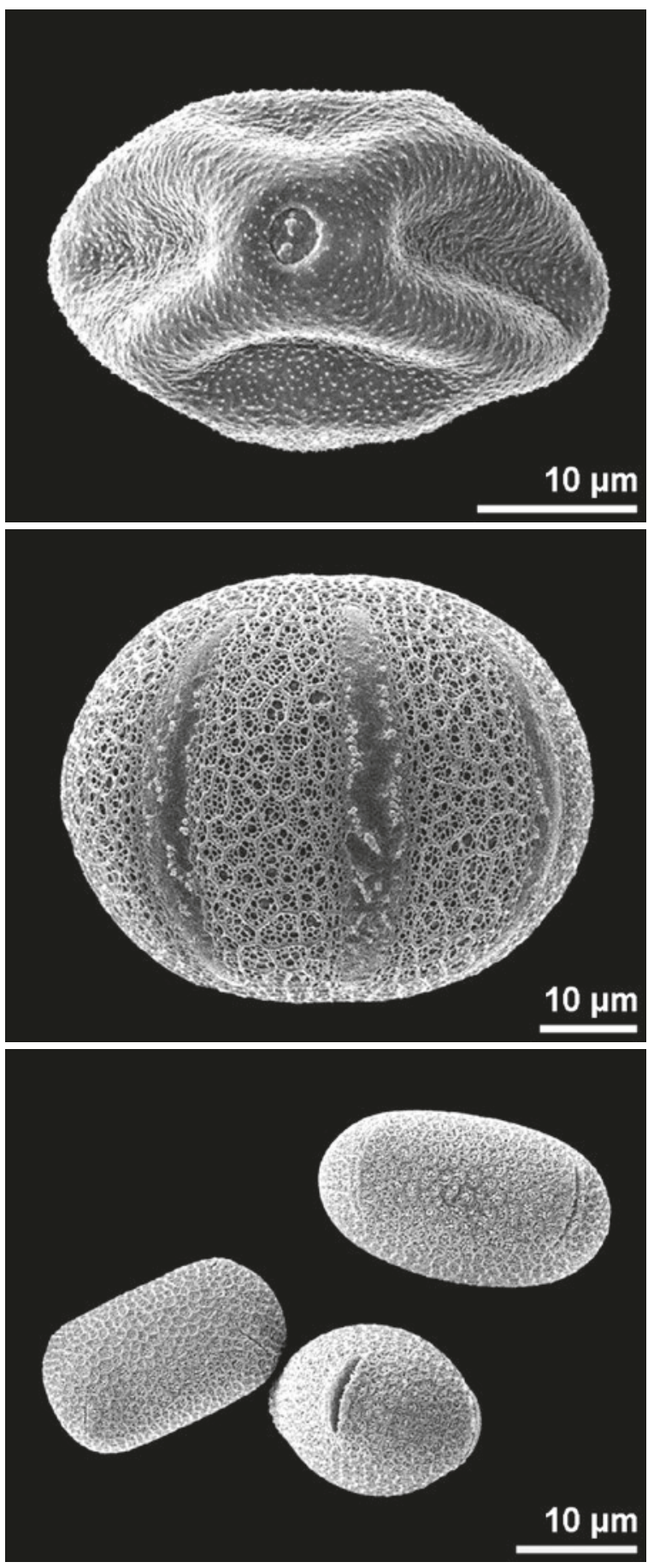

- Corylus avellana, Betulaceae triporate, equatorial view, dry pollen

Salvia argentea, Lamiaceae hexacolpate, equatorial view

Impatiens glandulifera, Balsaminaceae tetracolpate 

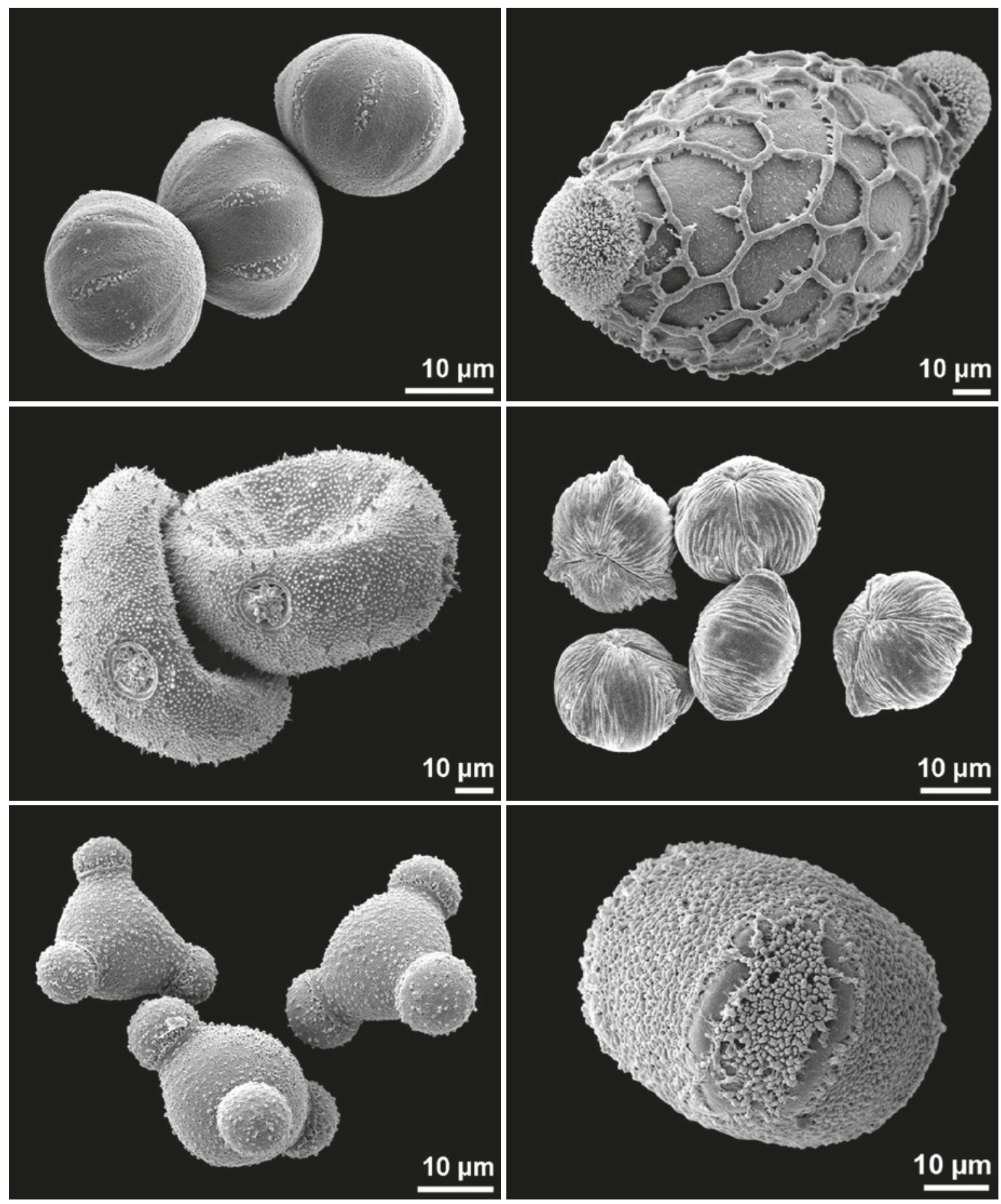

- Sarracenia alata, Sarraceniaceae

- Hymenocallis tubiflora, Amaryllidaceae stephanocolporate sulcate, proximal polar view

- Cuphea procumbens, Lythraceae tricolporate, dry pollen

Roridula gorgonias, Roridulaceae tricolpate, equatorial view 

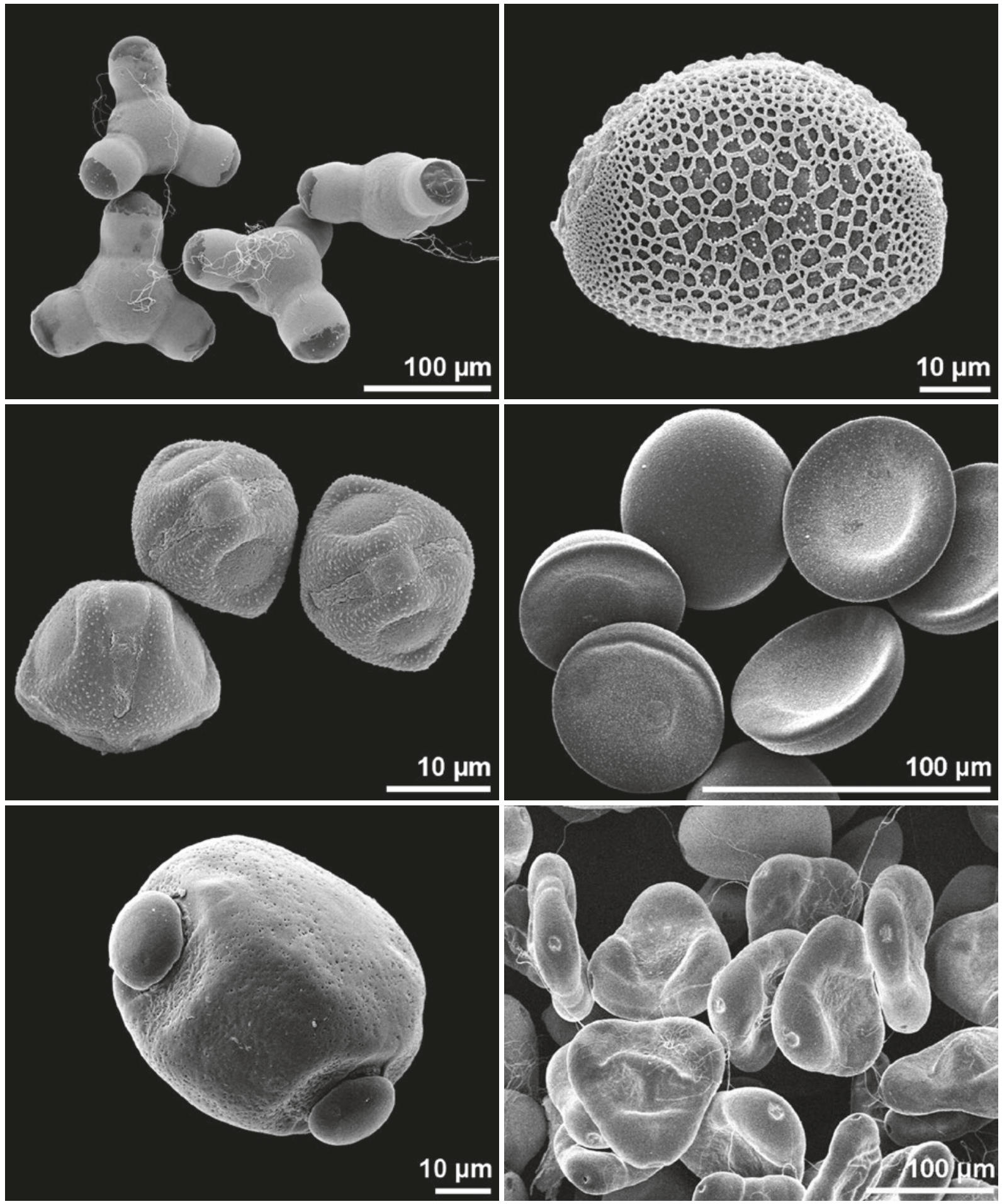

- Clarkia unguiculata, Onagraceae

- Vriesea neoglutinosa, Bromeliaceae triporate, pollen with viscin threads sulcate, equatorial view

Acicarpha tribuloides, Calyceraceae

Heliconia sp., Heliconiaceae tricolporate

ulcerate, dry pollen

Clarkia purpurea, Onagraceae

tricolporate, oblique equatorial view

triporate, dry pollen

170 | ILLUSTRATED POLLEN TERMS 


\section{$\mathrm{P} /$ E-ratio, isodiametric}

isodiametric: pollen grain with a polar axis equal to the equatorial diameter
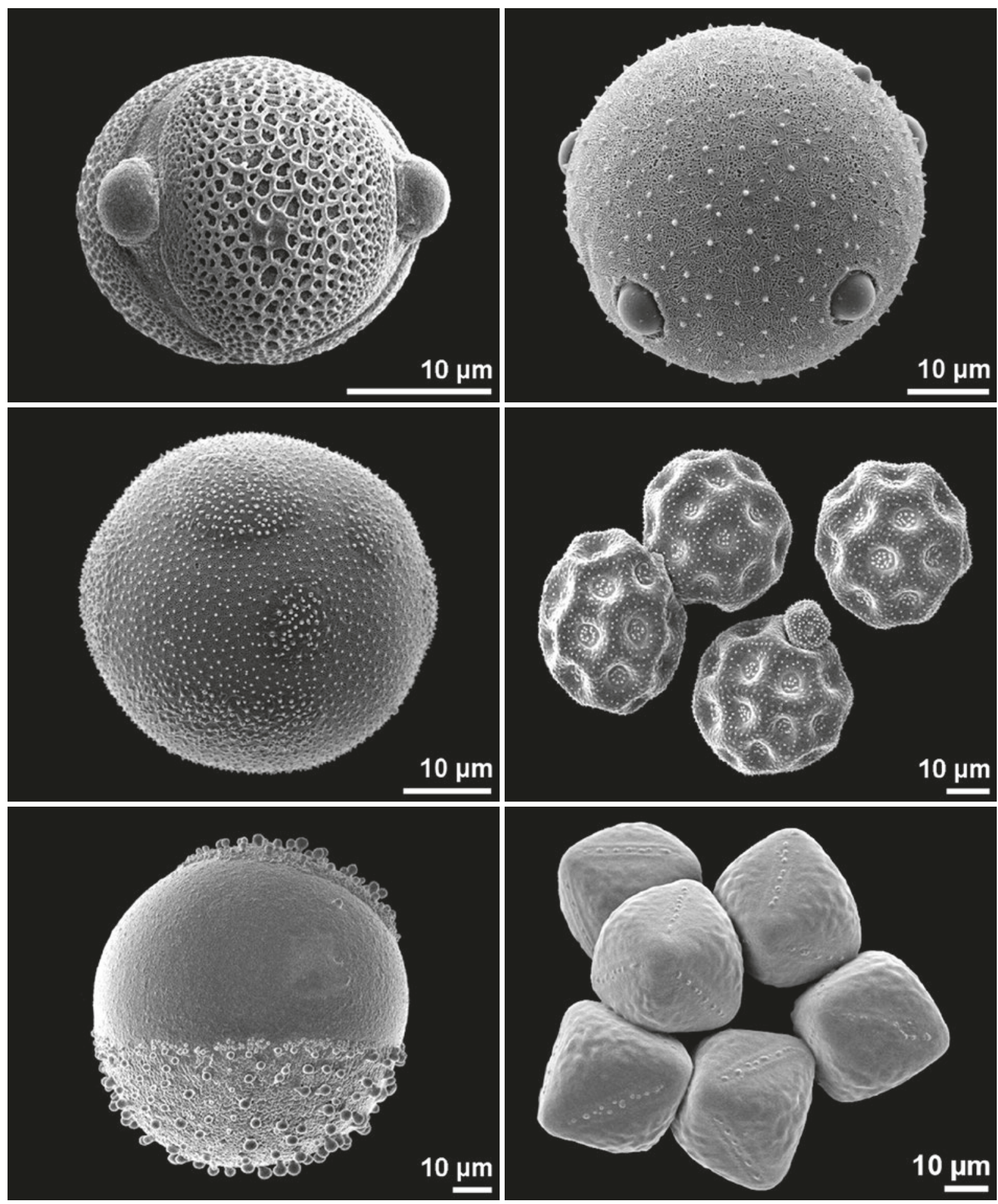

- Parnassia palustris, Celastraceae spheroidal, tricolporate, equatorial view

- Campanula fenestrellata, Campanulaceae spheroidal, stephanoporate, oblique polar view

- Roemeria hybrida, Papaveraceae spheroidal, pantoporate

- Silene nutans, Caryophyllaceae polygonal, pantoporate, dry pollen

Sarcocapnos enneaphylla, Papaveraceae hexacolpate 

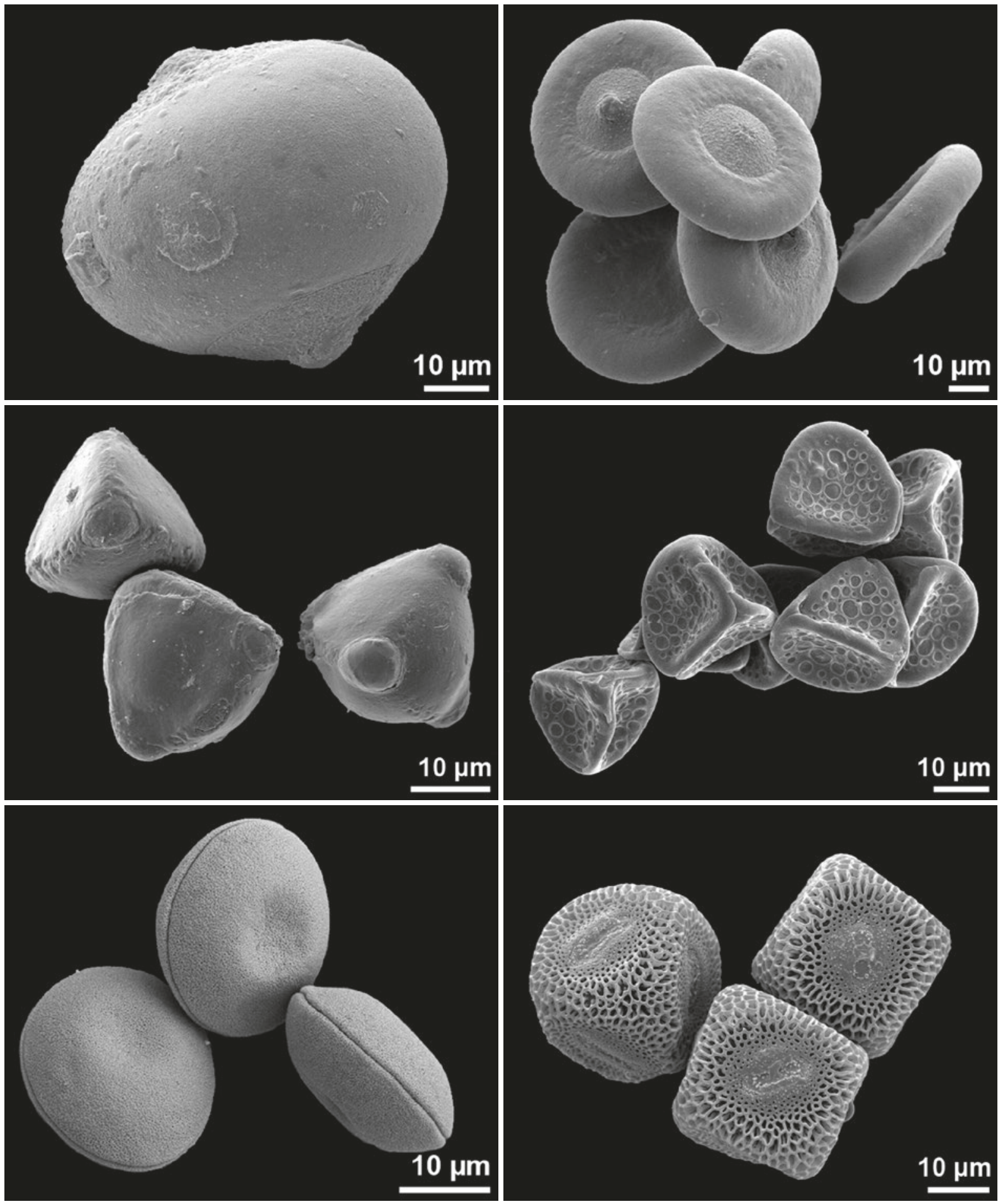

Whitfieldia lateritia, Acanthaceae

Whitfieldia lateritia, Acanthaceae diporate dry pollen

Thesium arvense, Santalaceae
triradiate colpi, dry pollen

- Schoepfia sch

Basella alba, Basellaceae

Pedicularis gyroflexa, Orobanchaceae

n ring-like aperture, dry pollen

- hexacolpate

172 | ILLUSTRATED POLLEN TERMS 


\section{P/E-ratio, prolate}

prolate: pollen grain with a polar axis longer than the equatorial diameter

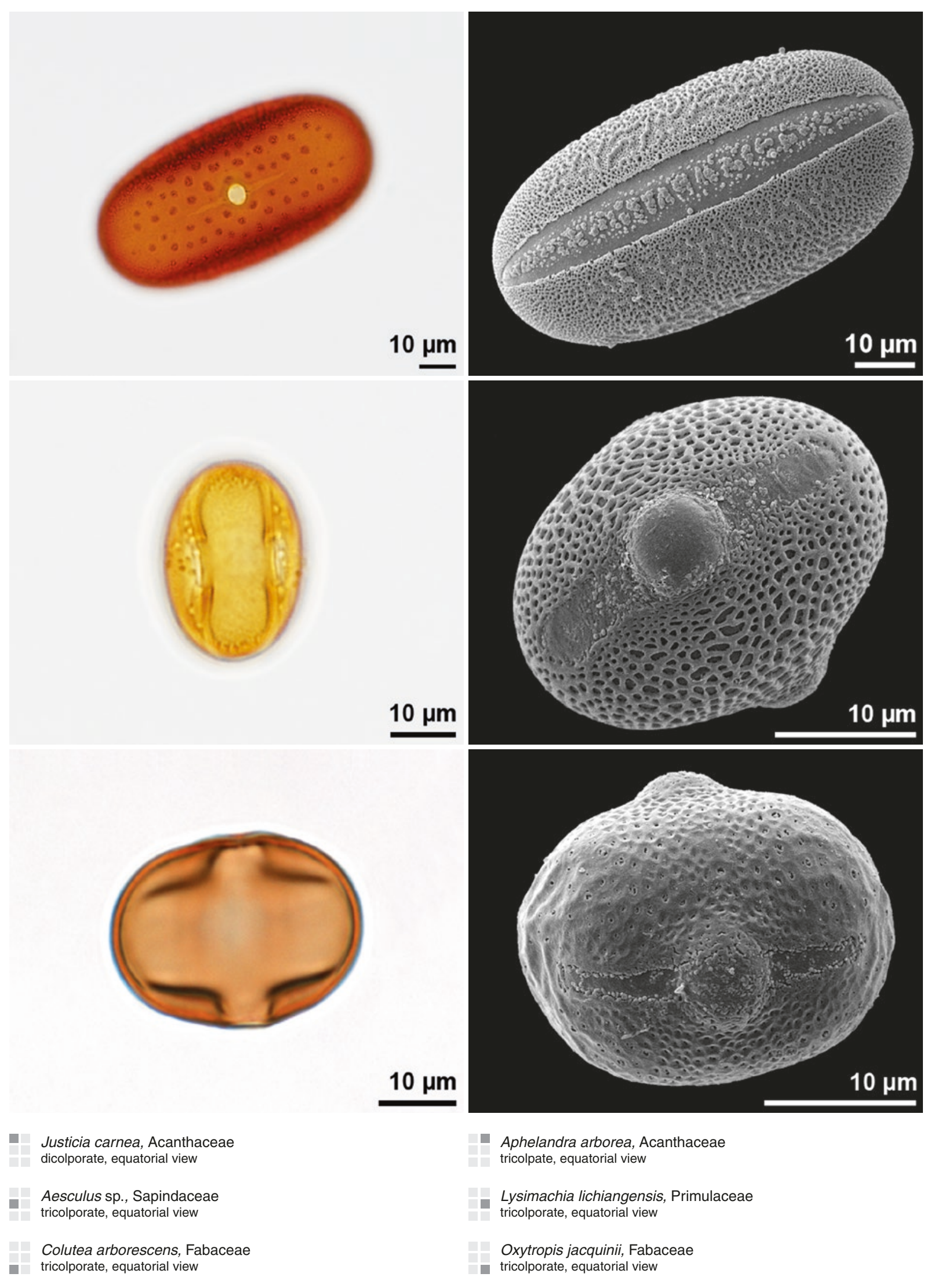



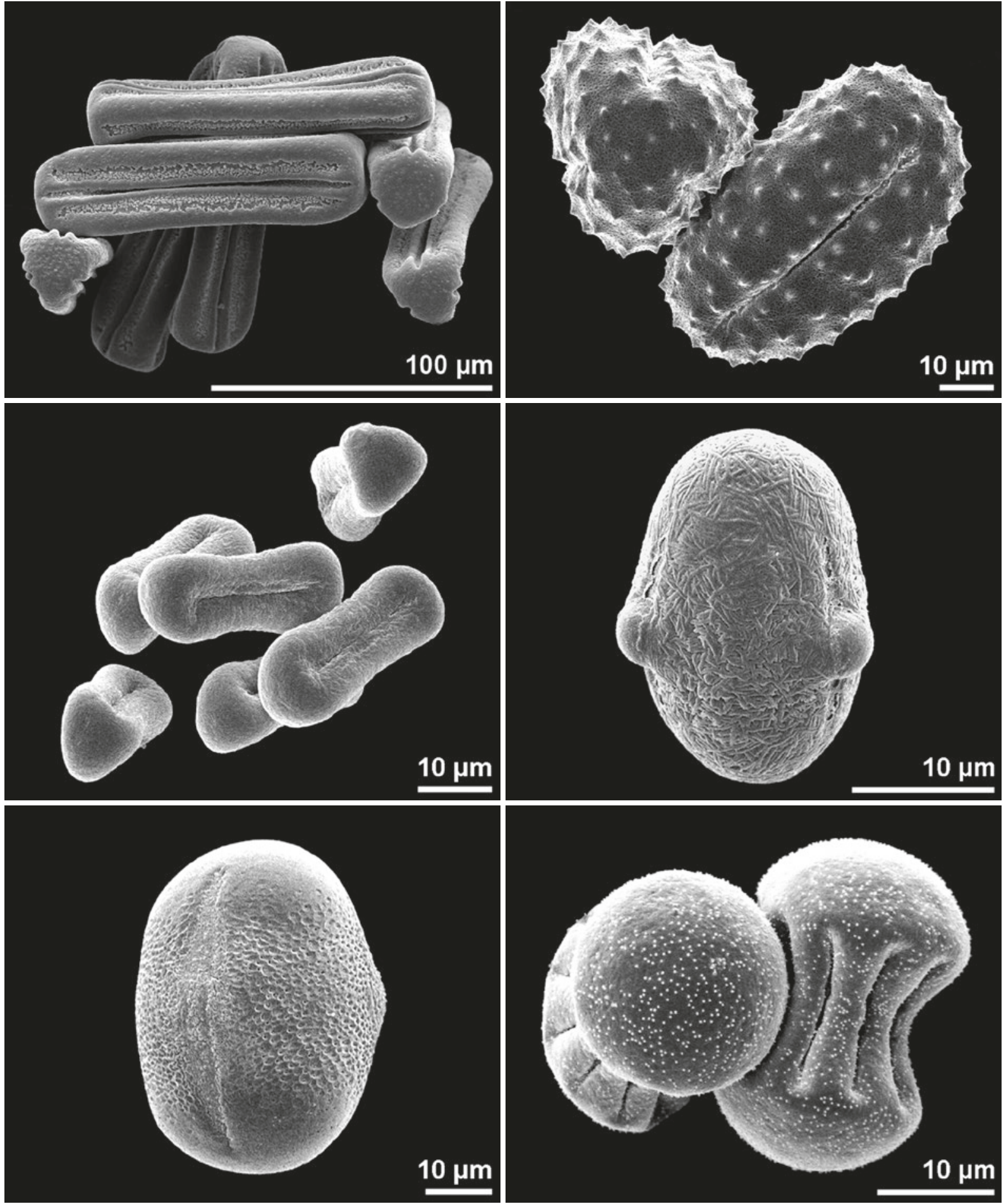

- Crossandra flava, Acanthaceae

- Jurinea mollis, Asteraceae tricolpate, dry pollen tricolporate, dry pollen Peucedanum cervaria, Apiaceae
tricolporate, equatorial view

- Torilis arvensis, Apiaceae

Symphytum officinale, Boraginaceae

Astragalus onobrychis, Fabaceae

- tricolporate, equatorial view

stephanocolporate, dry pollen 

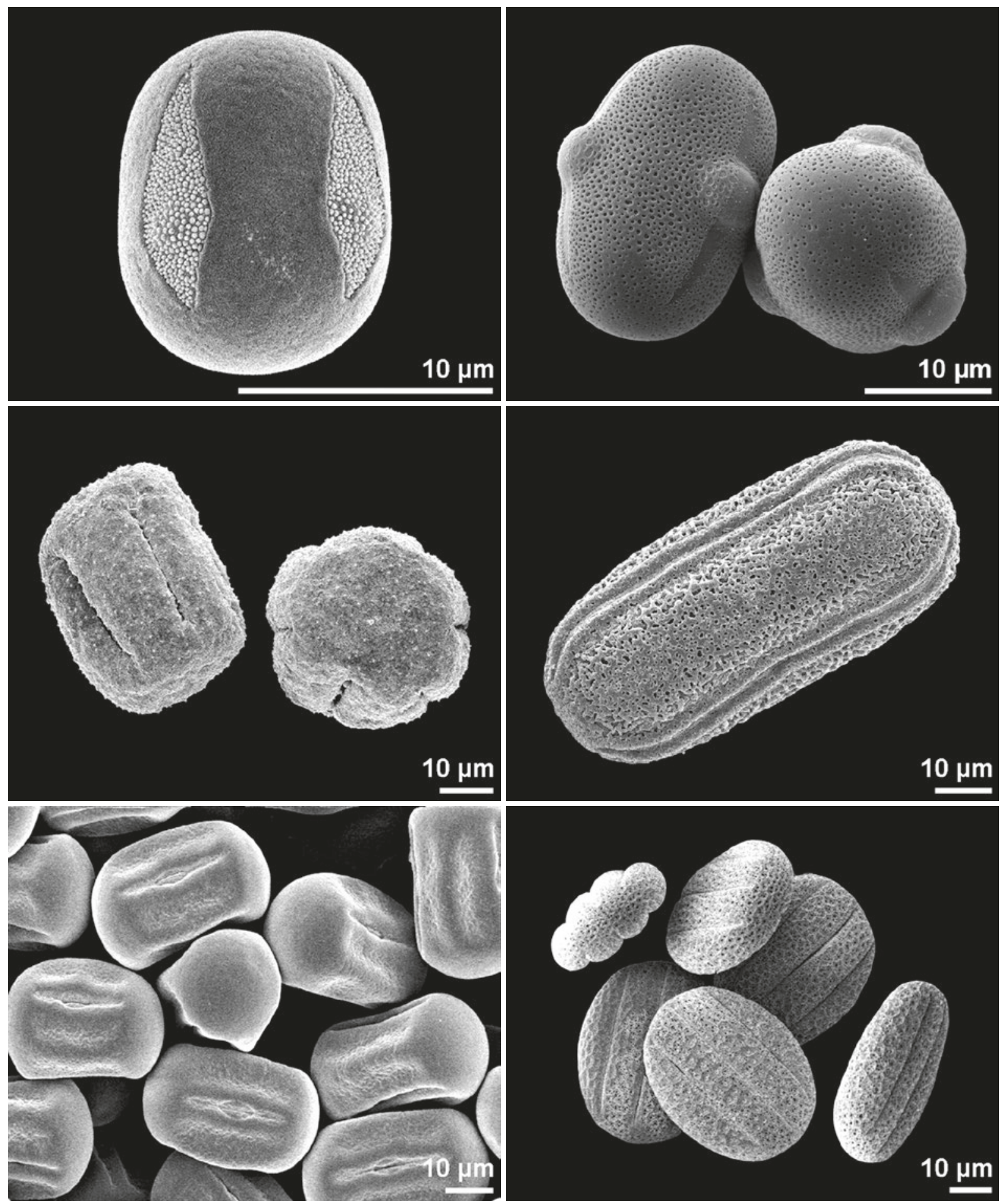

- Buglossoides purpurocaerulea, Boraginaceae

- Vitaliana primuliflora, Primulaceae tetracolporate, equatorial view tricolporate
- Stenandrium guineense, Acanthaceae
tricolpate, equatorial view
Salvia sclarea, Lamiaceae
- hexacolpate, dry pollen 


\section{isopolar}

pollen grain with identical proximal and distal faces
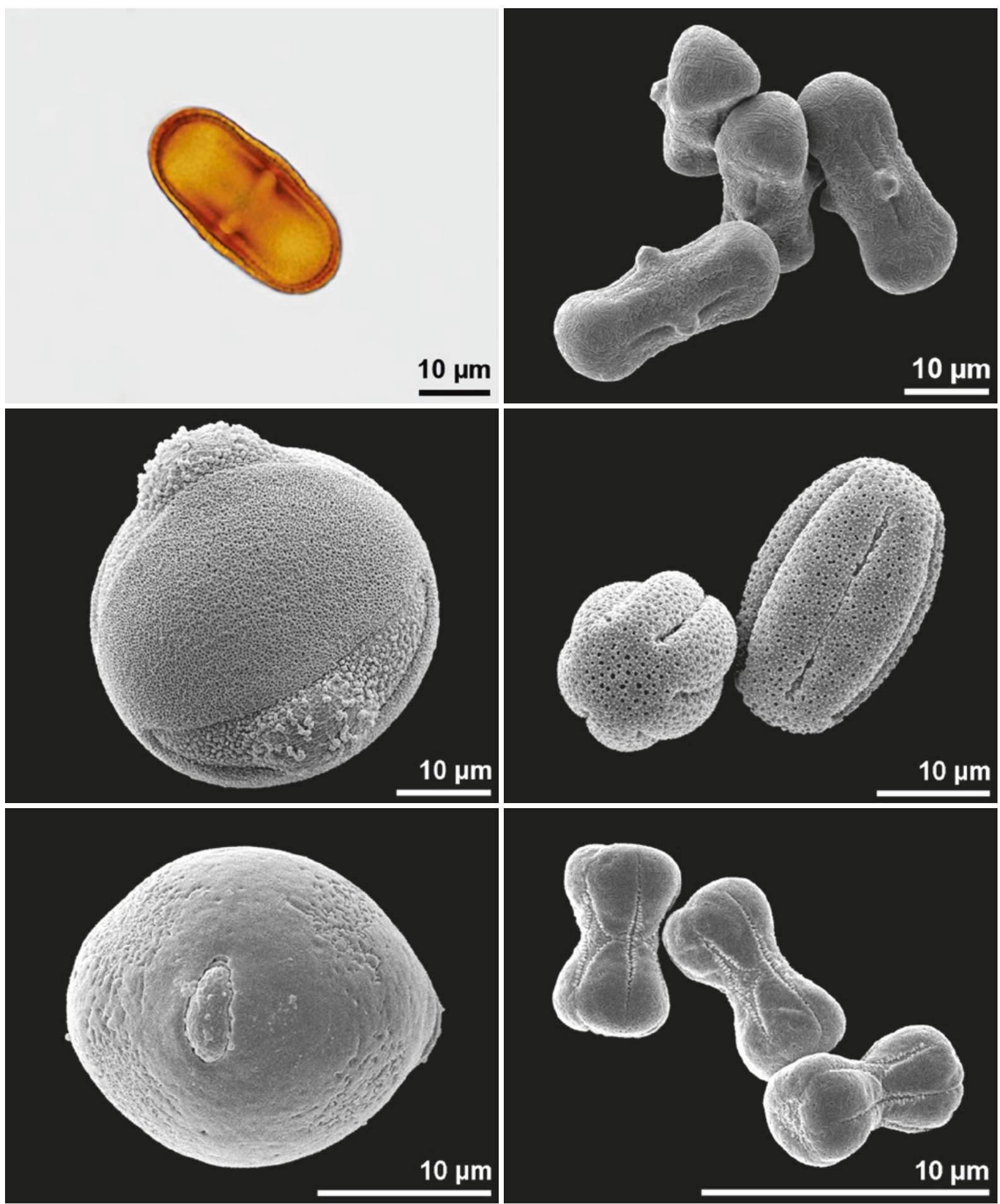

\footnotetext{
1. Apiaceae

equatorial view
Columnea magnifica, Gesneriaceae equatorial view

Monotropa hypopitys, Ericaceae - equatorial view
}

Bifora radians, Apiaceae Asperula tinctoria, Rubiaceae
dry pollen

Myosotis scorpioides, Boraginaceae

dry pollen 

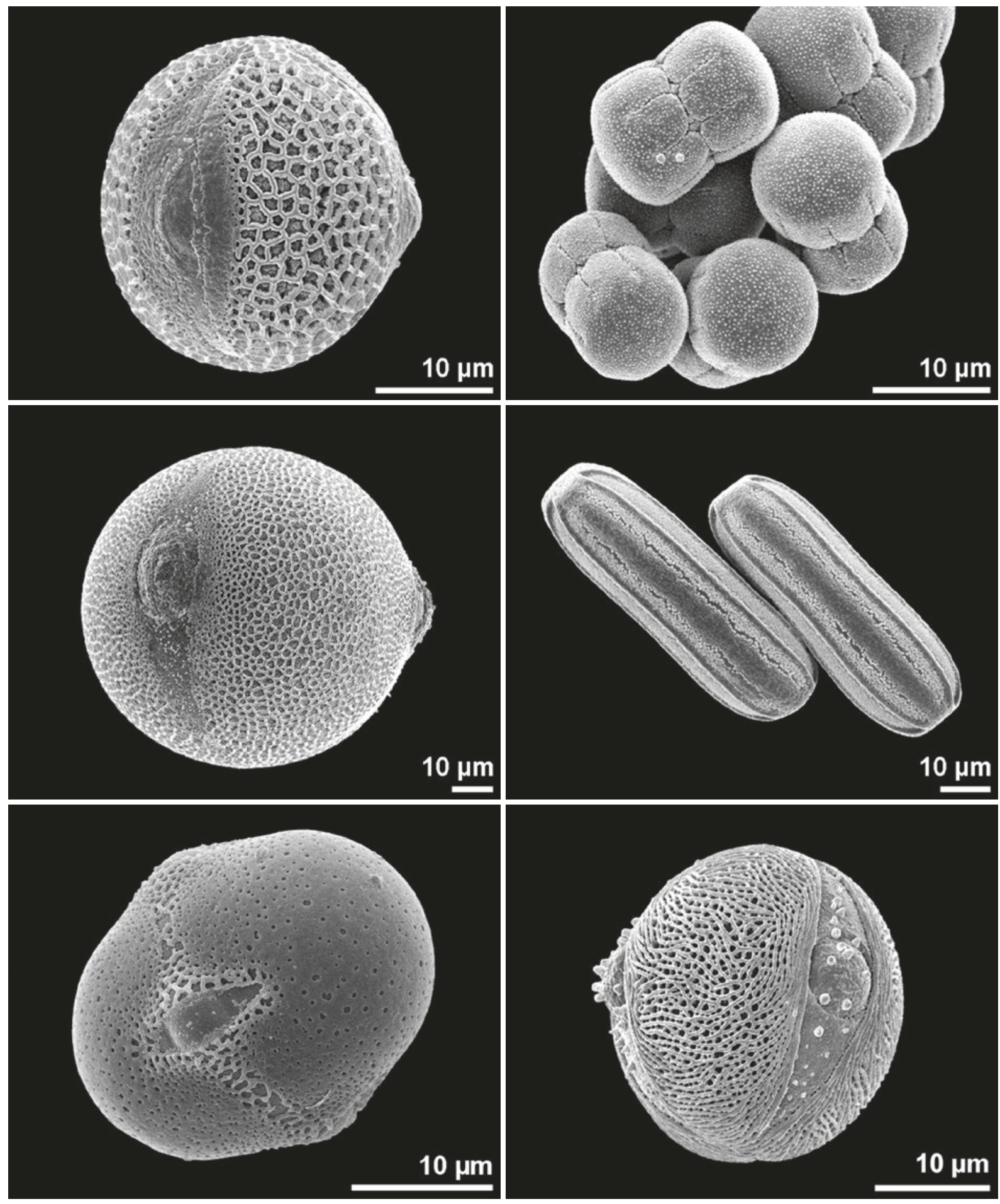

- Viburnum tinus, Adoxaceae

- Cerinthe minor, Boraginaceae equatorial view dry pollen

- Crossandra flava, Acanthaceae

Luffa cylindrica, Cucurbitaceae equatorial view

equatorial view

Pulmonaria angustifolia, Boraginaceae

- equatorial view

Aesculus flava, Sapindaceae

- equatorial view 


\section{heteropolar}

pollen grain with different proximal and distal faces
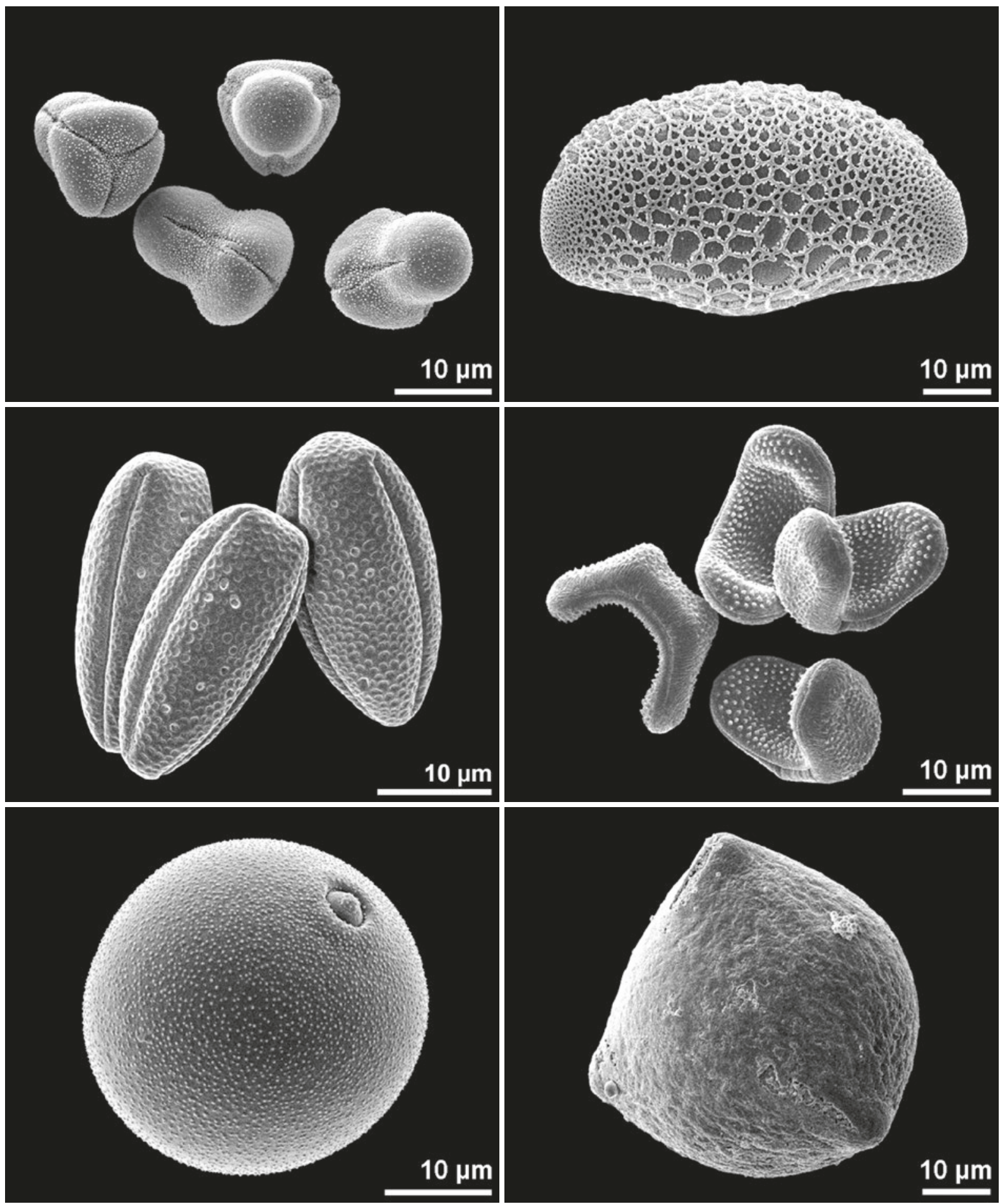

- Onosma visianii, Boraginaceae

Billbergia seidelii, Bromeliaceae

dry pollen

equatorial view

Chaenorhinum minus, Plantaginaceae

dry pollen

Limnanthes douglasii, Limnanthaceae dry pollen

Sesleria albicans, Poaceae

oblique view 

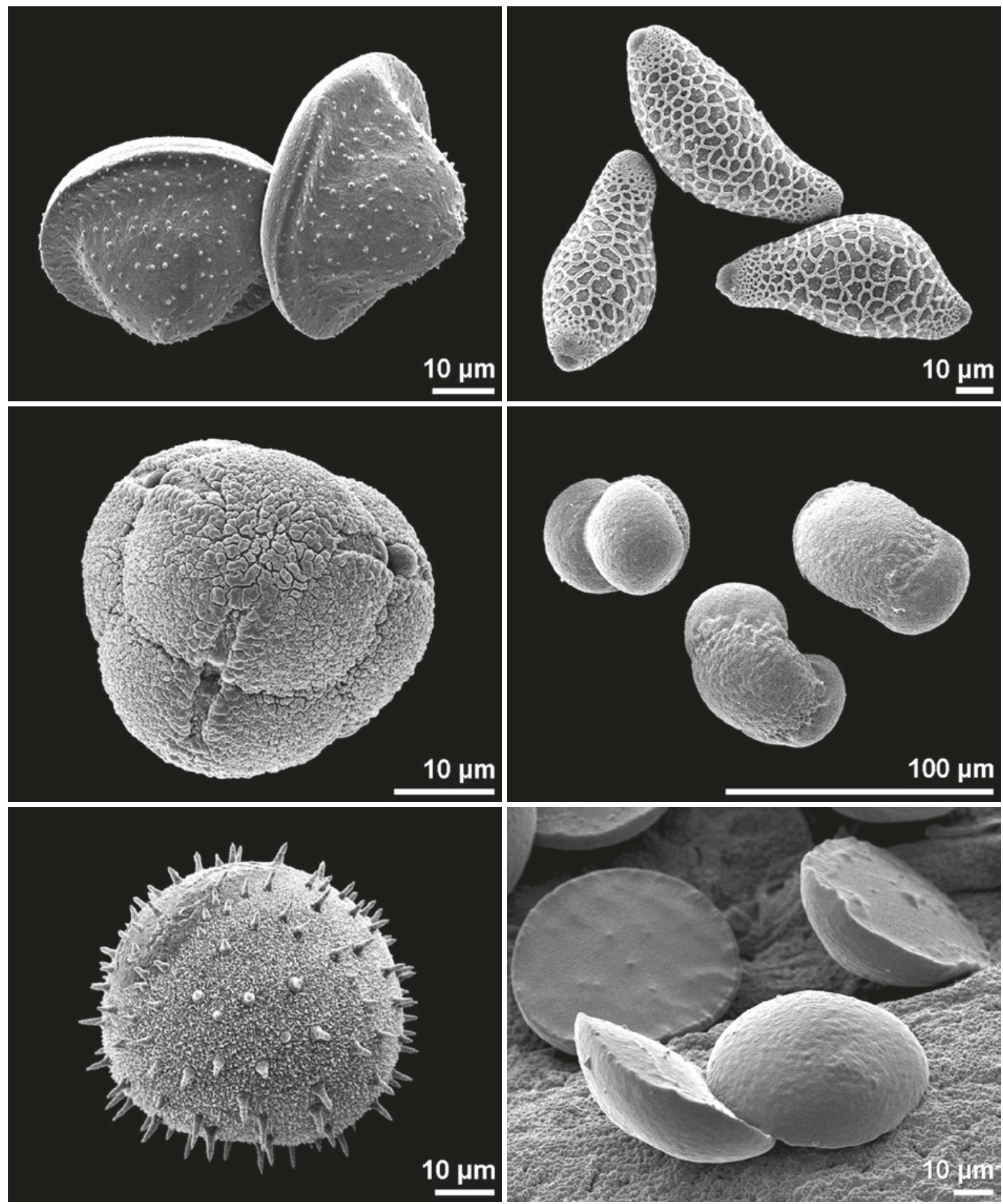

Heliconia sp., Heliconiaceae

- Quesnelia augusto-coburgii, Bromeliaceae dry pollen

Erica arborea, Ericaceae tetrad

- Pinus strobus, Pinaceae

Nuphar lutea, Nymphaeaceae

- equatorial view 

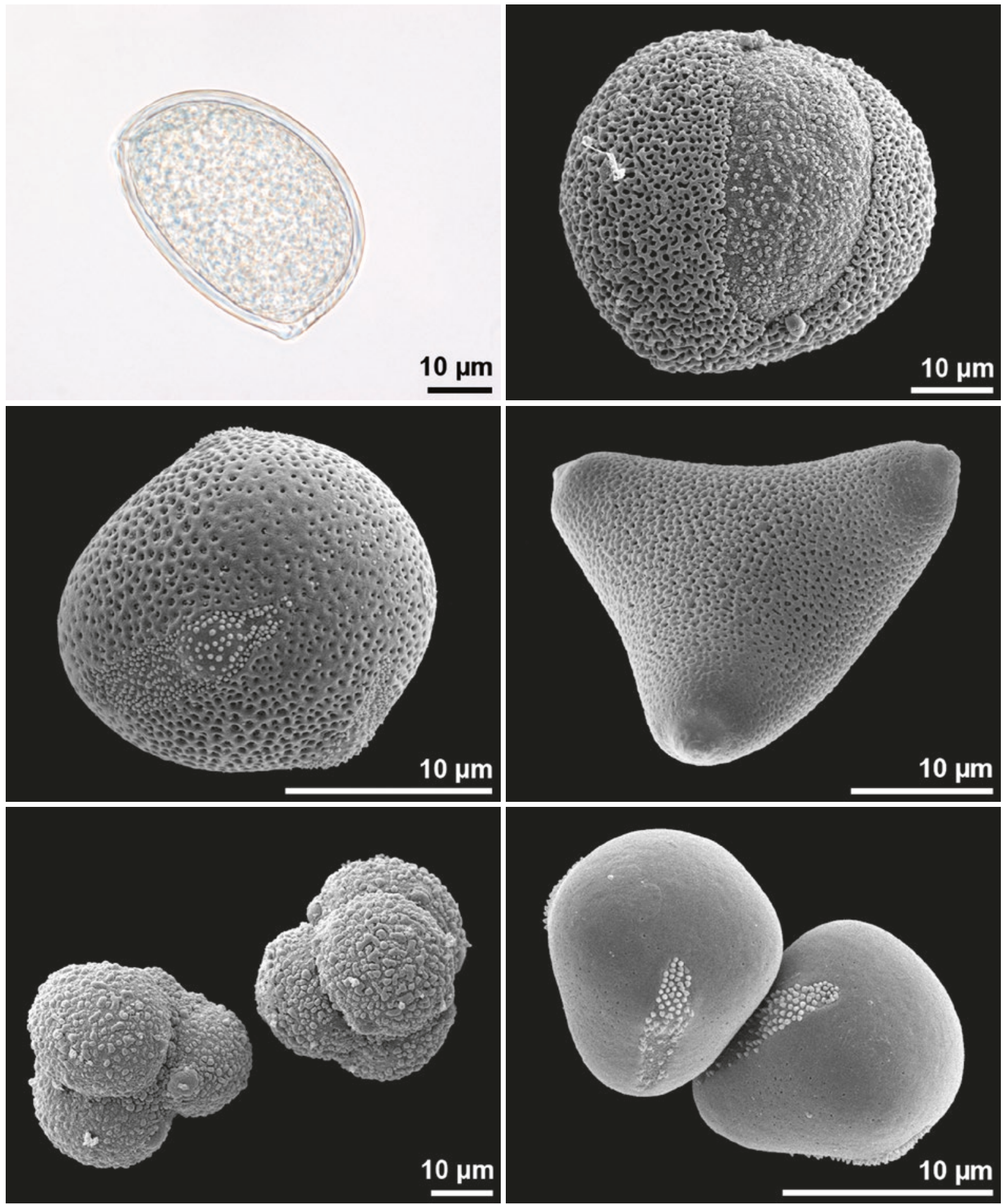

- Amorphophallus yunnanensis, Araceae hydrated, equatorial view

- Austrobaileya scandens, Austrobaileyaceae distal polar view

Adenanthos sericeus, Proteaceae

Echium italicum, Boraginaceae polar view

Calluna vulgaris, Ericaceae tetrads

Alkanna corcyrensis, Boraginaceae 


\section{shape}

3-dimensional form of a pollen grain in relation to the P/E-ratio
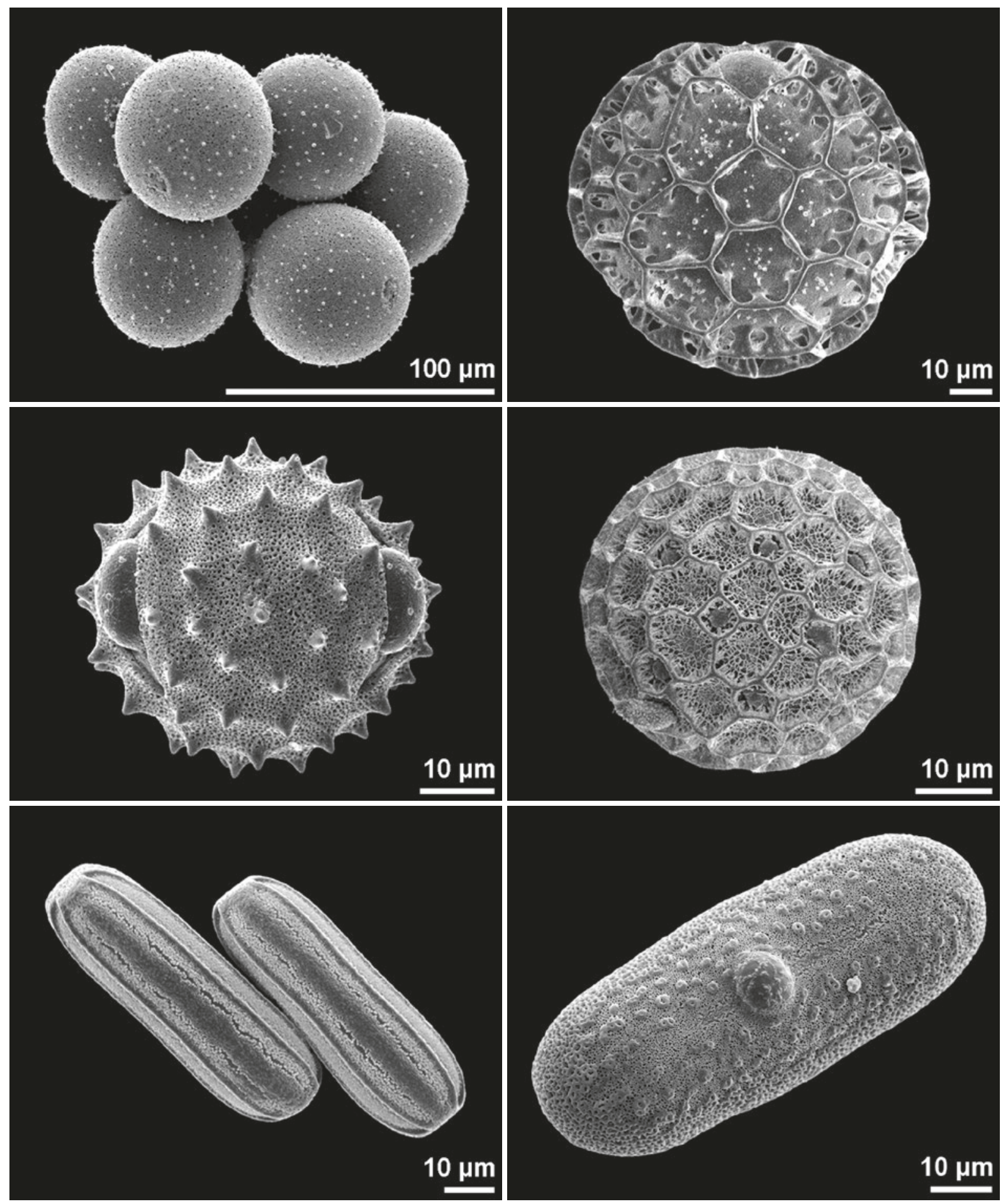

- Adansonia gregorii, Malvaceae

- Ruellia macrantha, Acanthaceae spheroidal

spheroidal, polar view

- Cirsium oleraceum, Asteraceae spheroidal, equatorial view Phlox paniculata, Polemoniaceae
spheroidal

Crossandra flava, Acanthaceae

- cylindric, equatorial view 

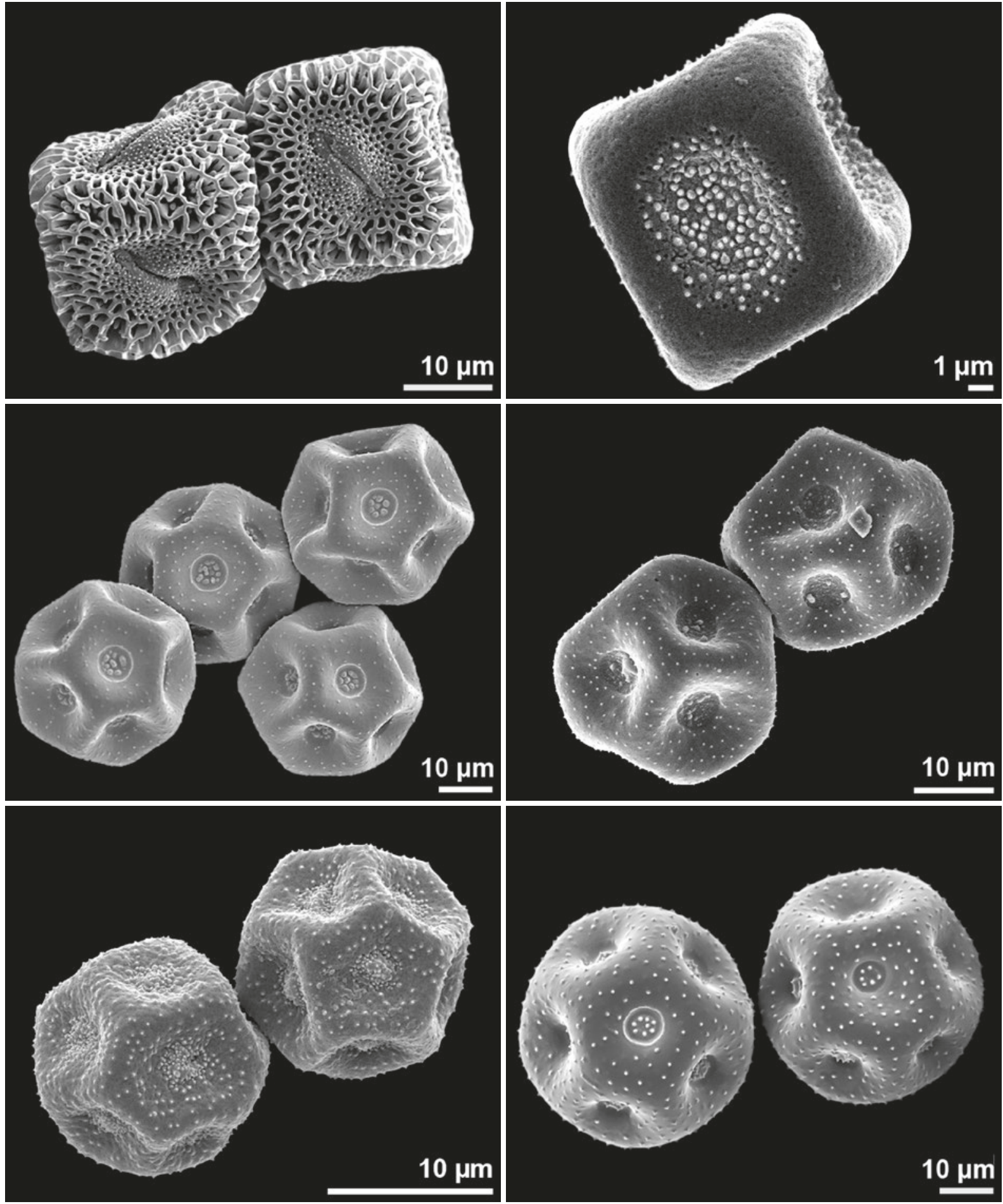

- Basella alba, Basellaceae cubical, dry pollen

Cerastium dubium, Caryophyllaceae polygonal, dry pollen

Paronychia polygonifolia, Caryophyllaceae

- polygonal, dry pollen
- Herniaria glabra, Caryophyllaceae cubical, dry pollen

Eremogone procera, Caryophyllaceae polygonal, dry pollen

Paronychia polygonifolia, Caryophyllaceae

polygonal, dry pollen 

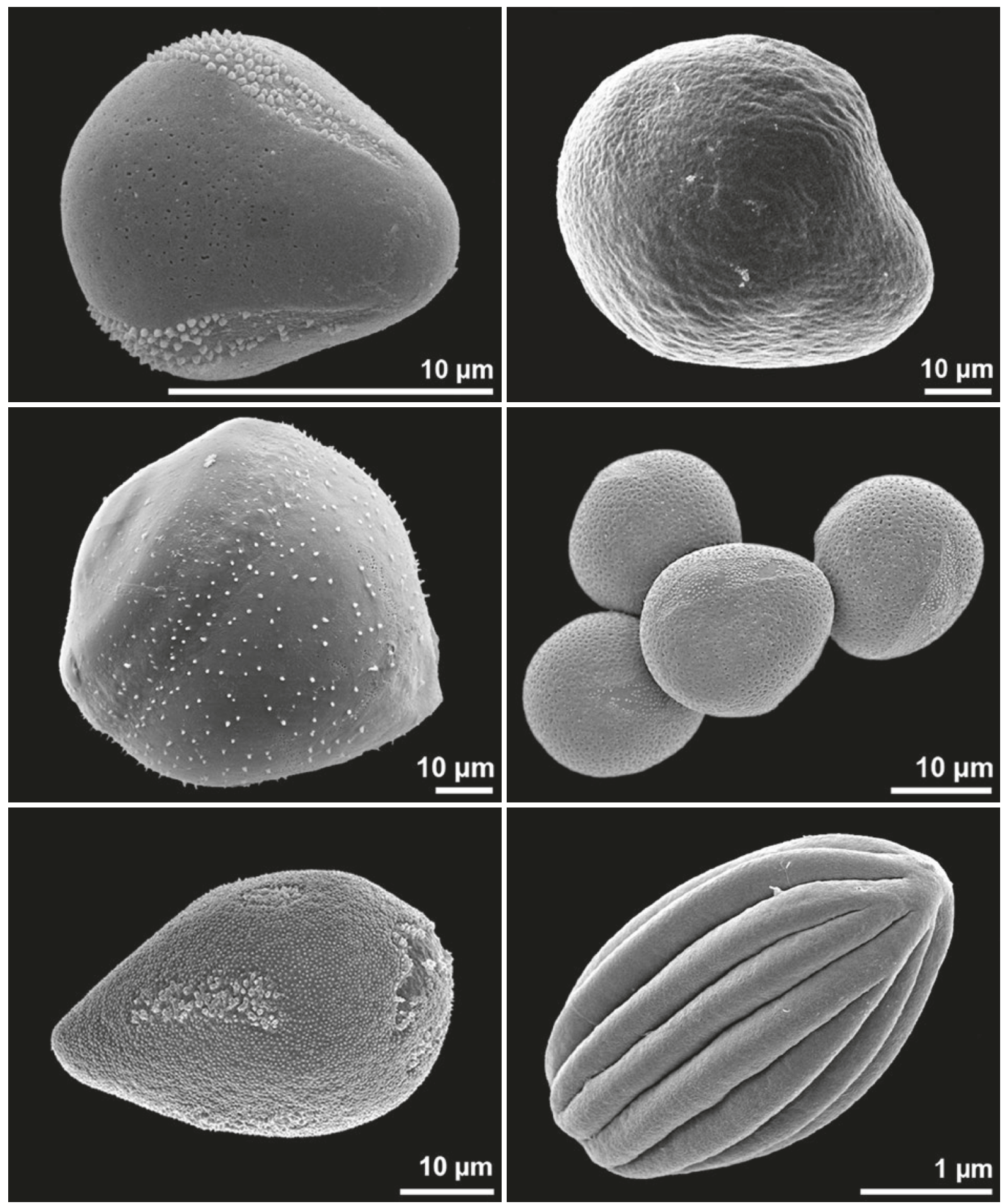

- Alkanna corcyrensis, Boraginaceae

- Montrichardia arborescens, Araceae pear-shaped, equatorial view pear-shaped, equatorial view

- Heliconia rostrata, Heliconiaceae pear-shaped, equatorial view

Echium plantagineum, Boraginaceae

Cyperus longus, Cyperaceae

- pear-shaped, equatorial view, pseudomonad 

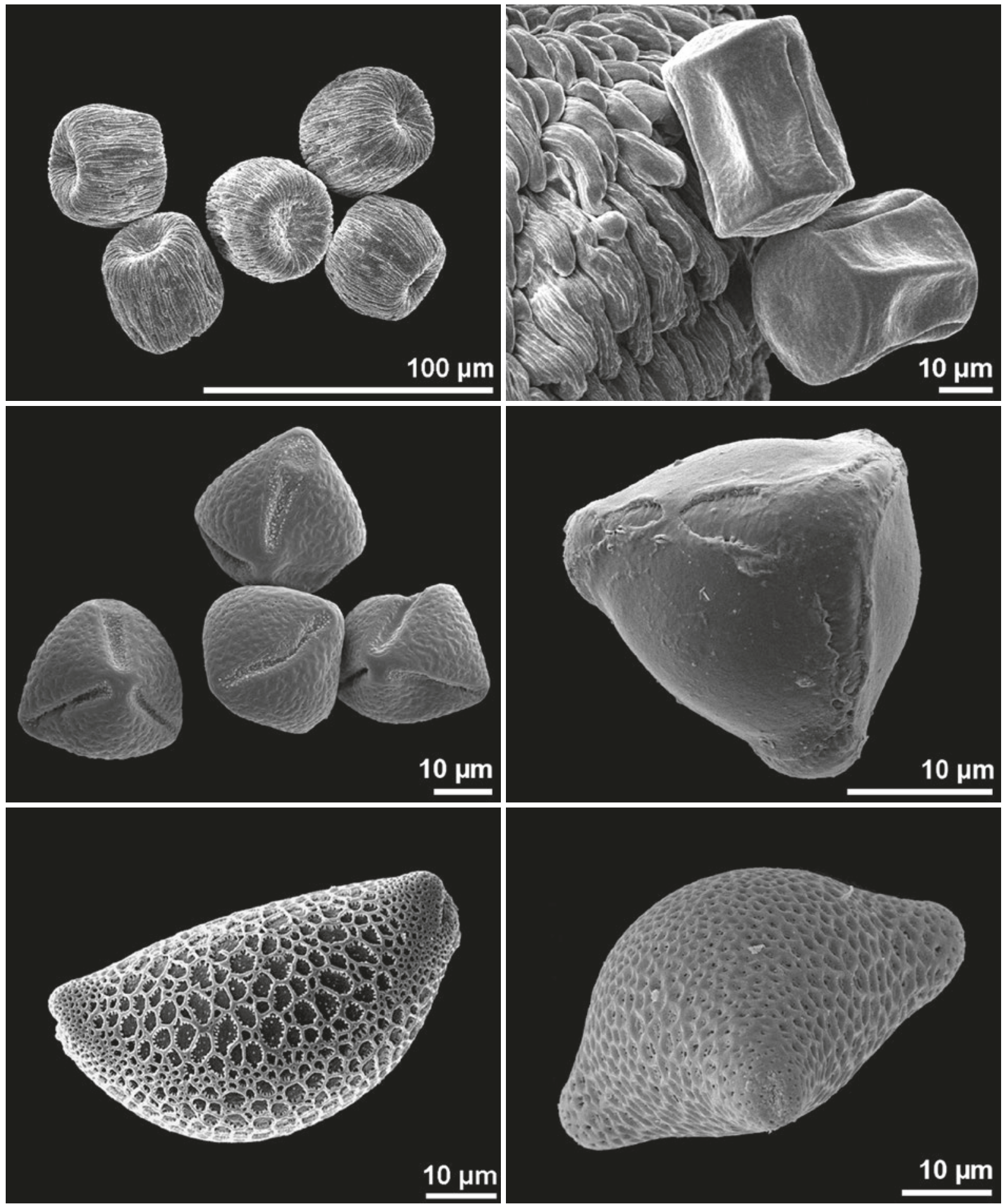

- Brugmansia suaveolens, Solanaceae barrel-shaped, dry pollen

- Corydalis cheilanthifolia, Papaveraceae triangular pyramid, dry pollen

Aechmea drakeana, Bromeliaceae wedge-shaped
- Anthyllis vulneraria, Fabaceae barrel-shaped, dry pollen

- Schoepfia schreberi, Schoepfiaceae triangular pyramid

Cardiospermum halicacabum, Sapindaceae convex triangular 

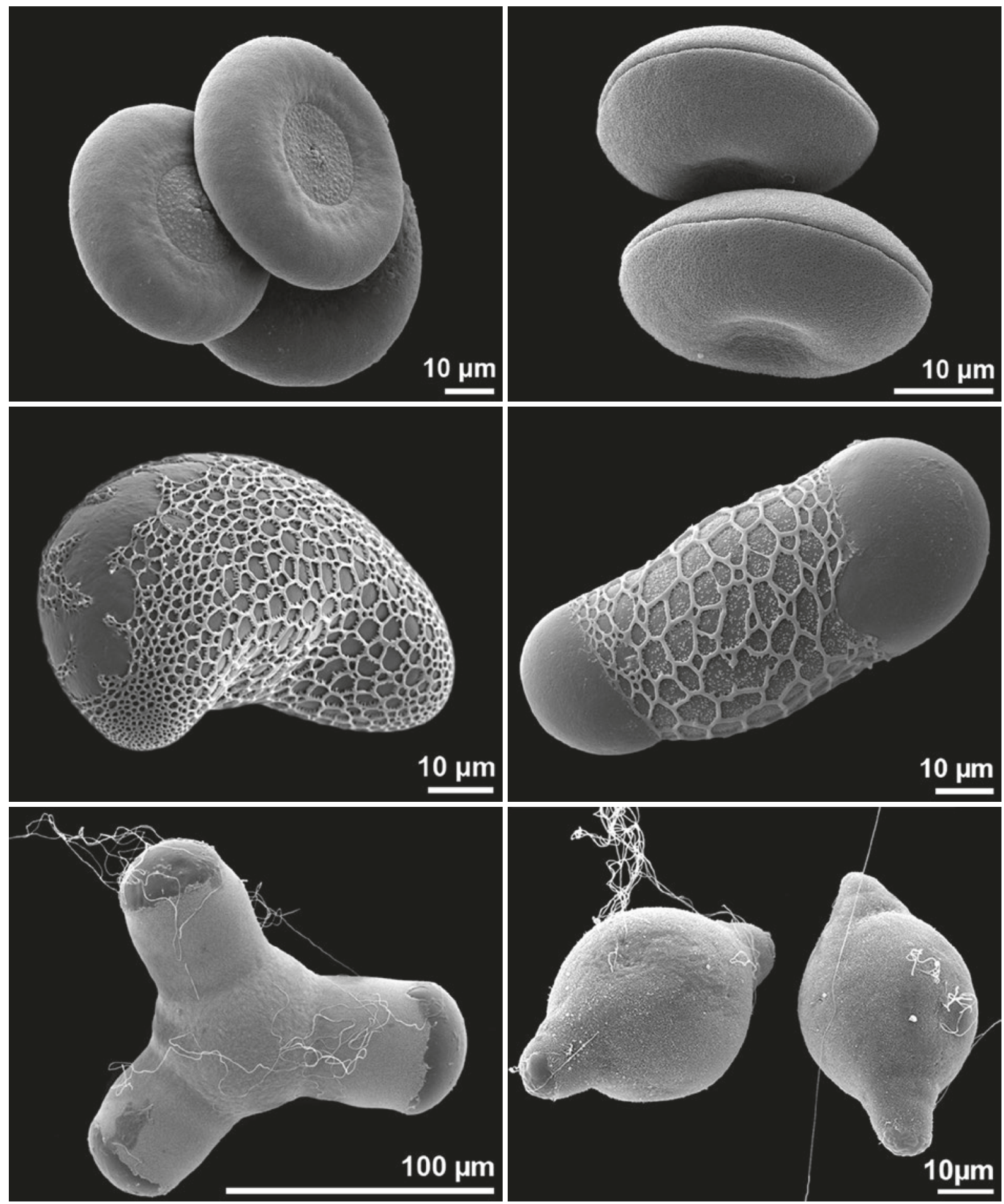

- Whitfieldia lateritia, Acanthaceae

- Pedicularis portenschlagii, Orobanchaceae disc-shaped, dry pollen disc-shaped, dry pollen

- Quesnelia imbricata, Bromeliaceae

Billbergia py
bean-shaped cylindric

Clarkia unguiculata, Onagraceae

- triangular star

\section{Fuchsia paniculata, Onagraceae} - lemon-shaped 

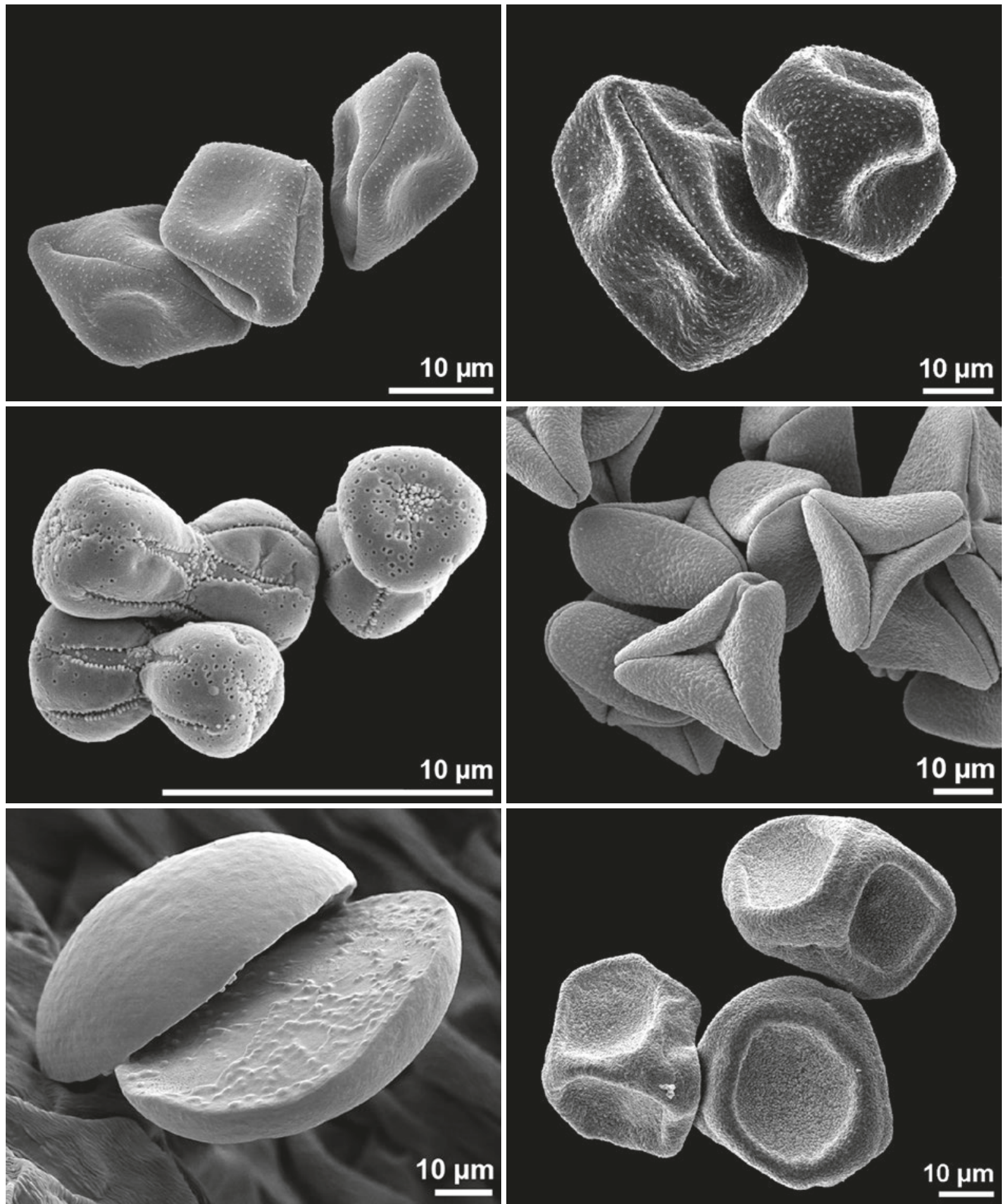

- Acicarpha tribuloides, Calyceraceae

Nicandra physalodes, Solanaceae dry pollen Loranthus europaeus, Loranthaceae
dry pollen

1. Myosotis alpestris, Boraginaceae

Sansevieria suffruticosa, Asparagaceae

Juncus jacquinii, Juncaceae

tetrad, monads cup-shaped, dry pollen 

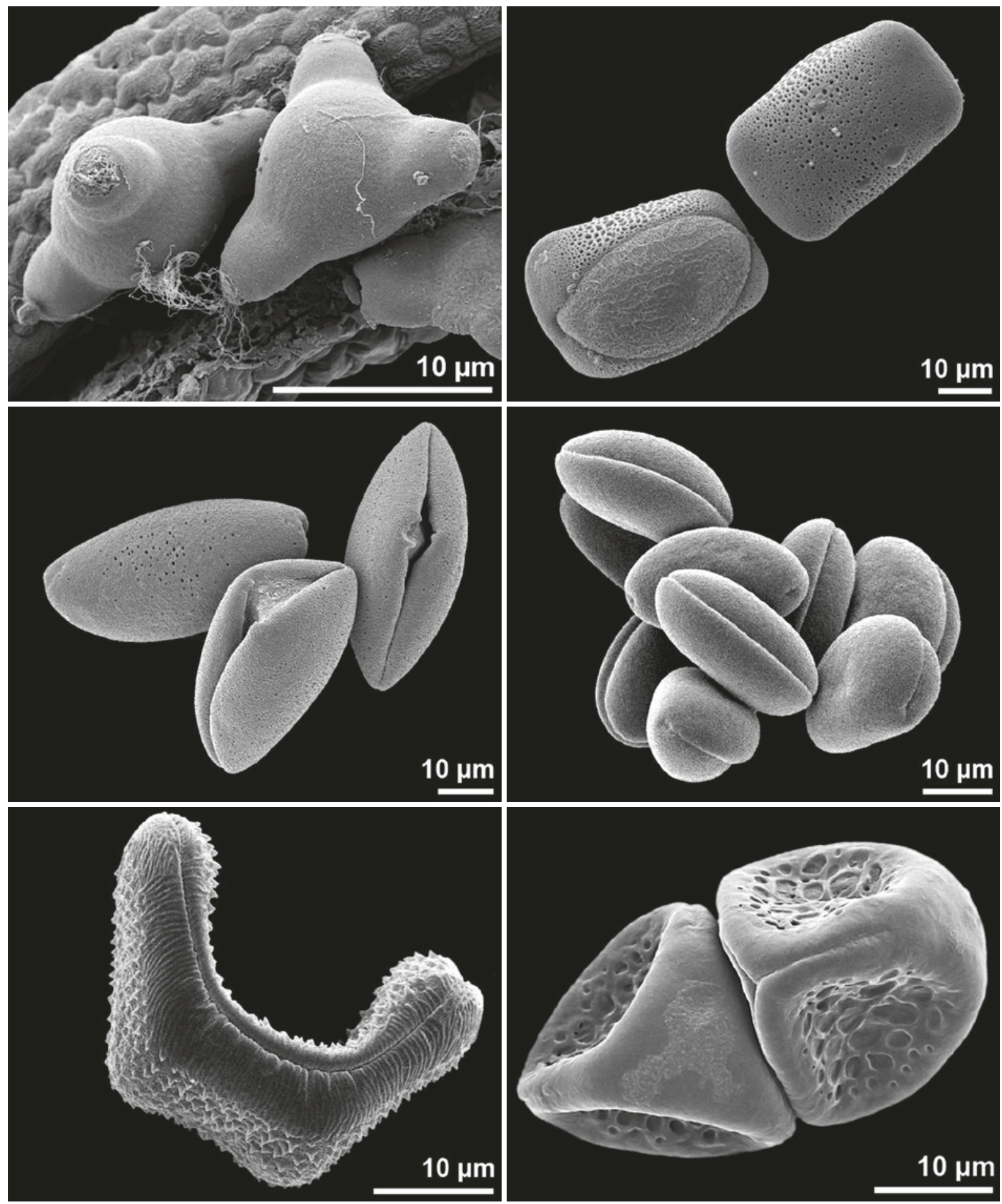

- Gaura lindheimeri, Onagraceae
Eremurus robustus, Xanthorrhoeaceae rounded cuboid
Galanthus nivalis, Amaryllidaceae
boat-shaped, dry pollen
Thesium dollineri, Santalaceae
- convex triangular pyramid, dry pollen
Hyacinthoides italica, Asparagaceae
boat-shaped, dry pollen
Limnanthes douglasii, Limnanthaceae
- U-shaped, dry pollen




\section{saccus/saccate, corpus}

saccus: exinous expansion forming an air sac corpus: body of a saccate pollen grain

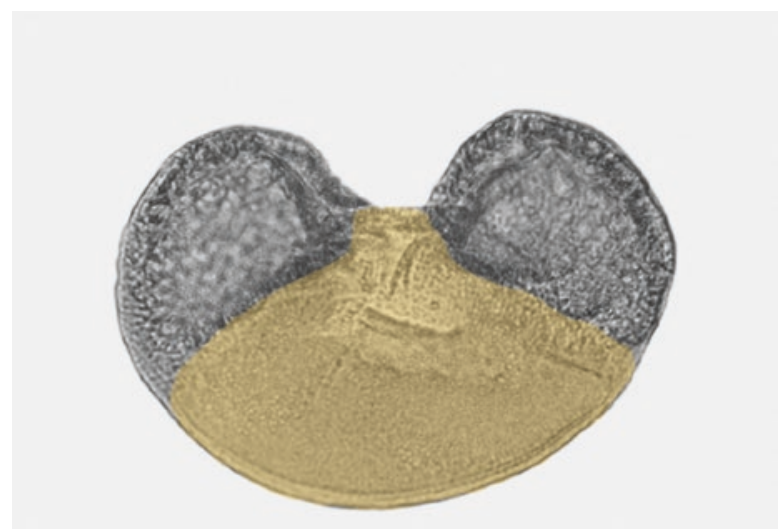

$10 \mu \mathrm{m}$
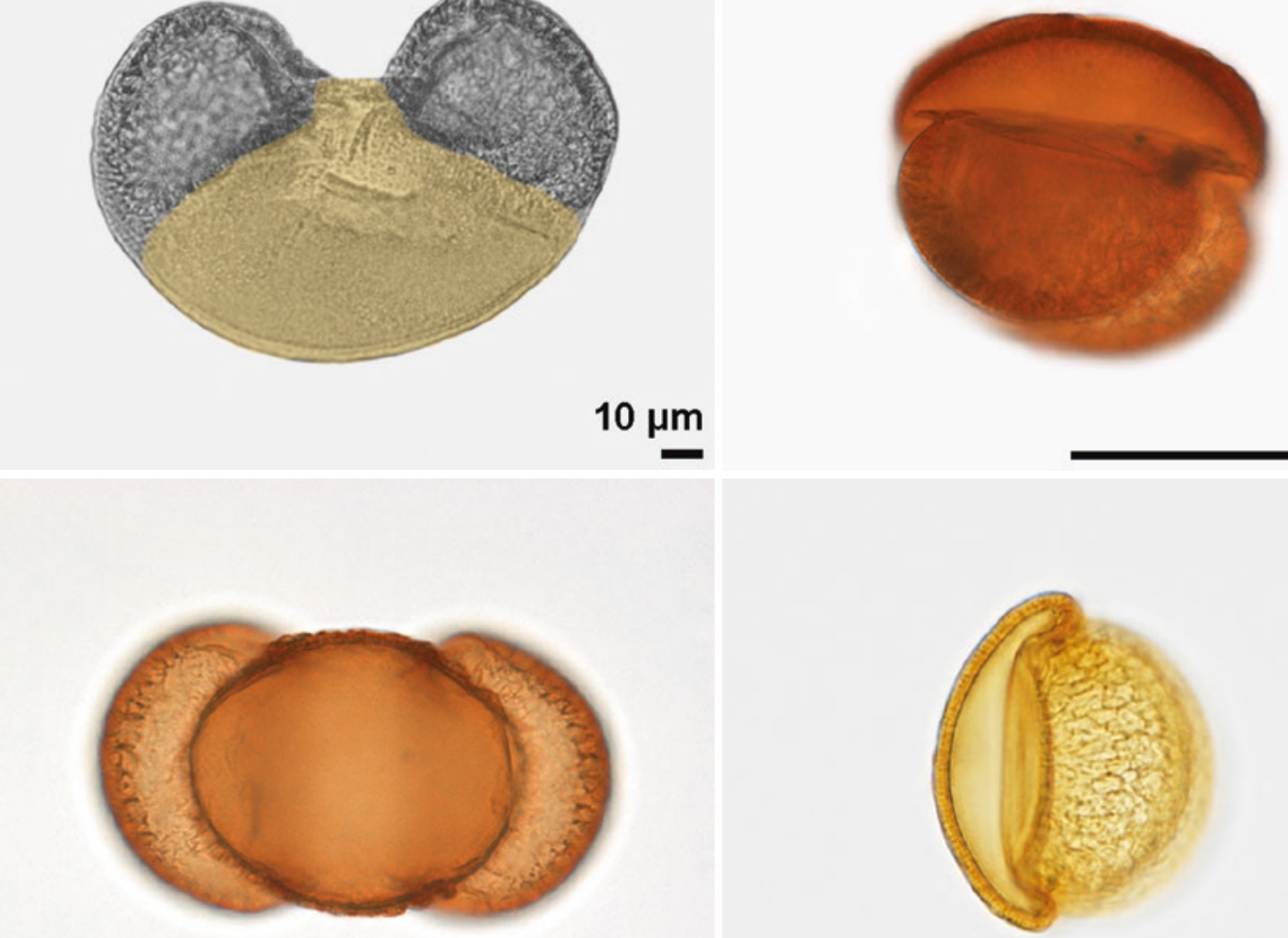

-

$100 \mu \mathrm{m}$

$10 \mu \mathrm{m}$

$10 \mu \mathrm{m}$

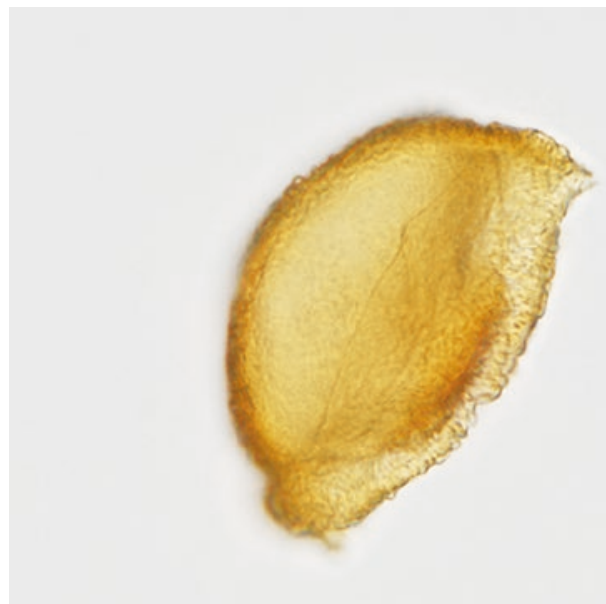

$10 \mu \mathrm{m}$

- Picea sp., Pinaceae

fossil, Miocene, China, corpus colored, equatorial view

- Pinus cembra, Pinaceae

proximal polar view

Tsuga canadensis, Pinaceae

- equatorial view

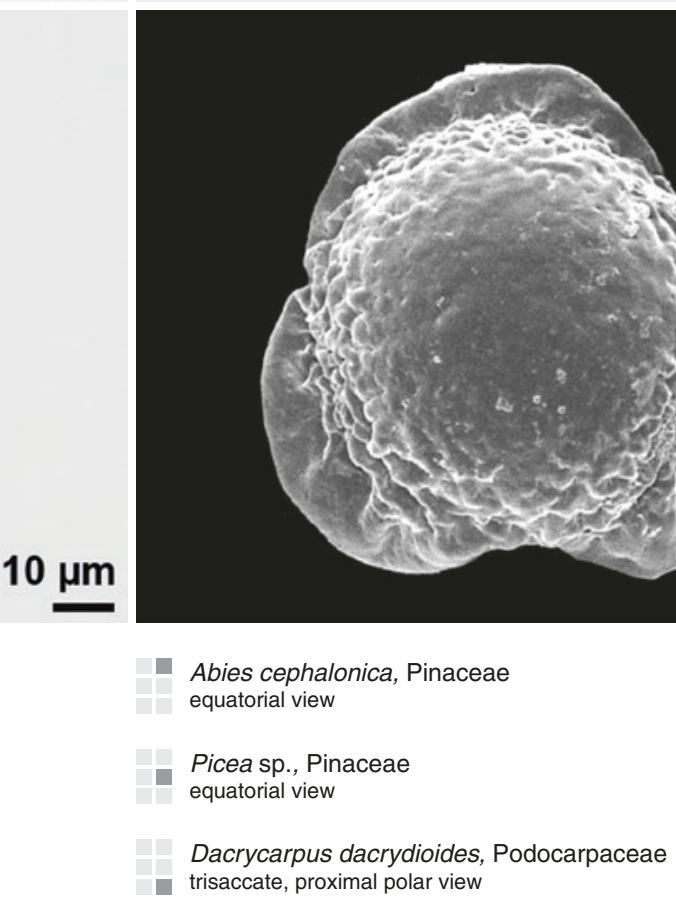




\section{saccus, monosaccate}

monosaccate: pollen grain with a single saccus
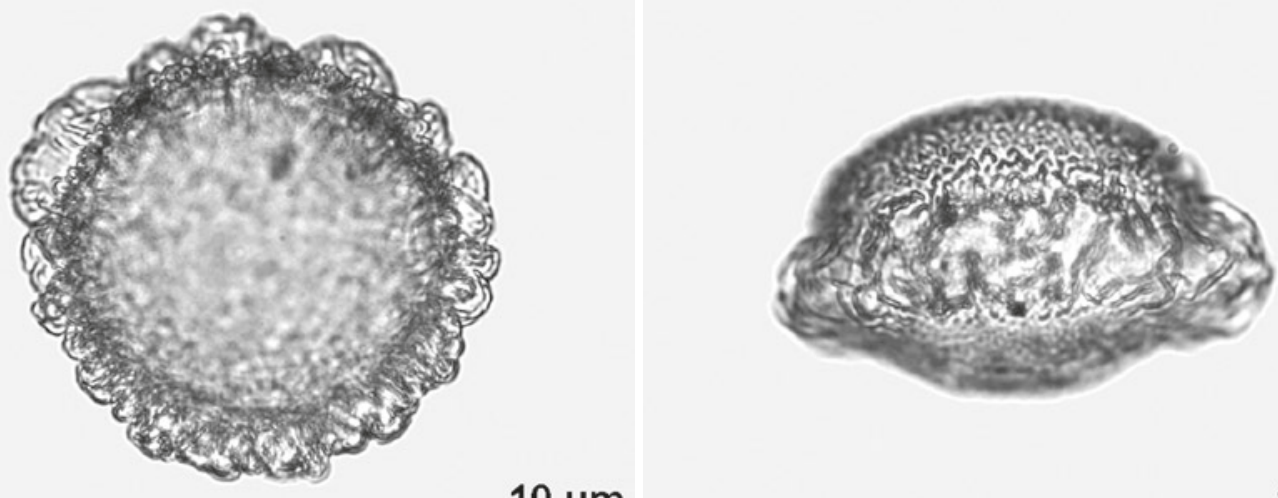

$10 \mu \mathrm{m}$

$10 \mu \mathrm{m}$
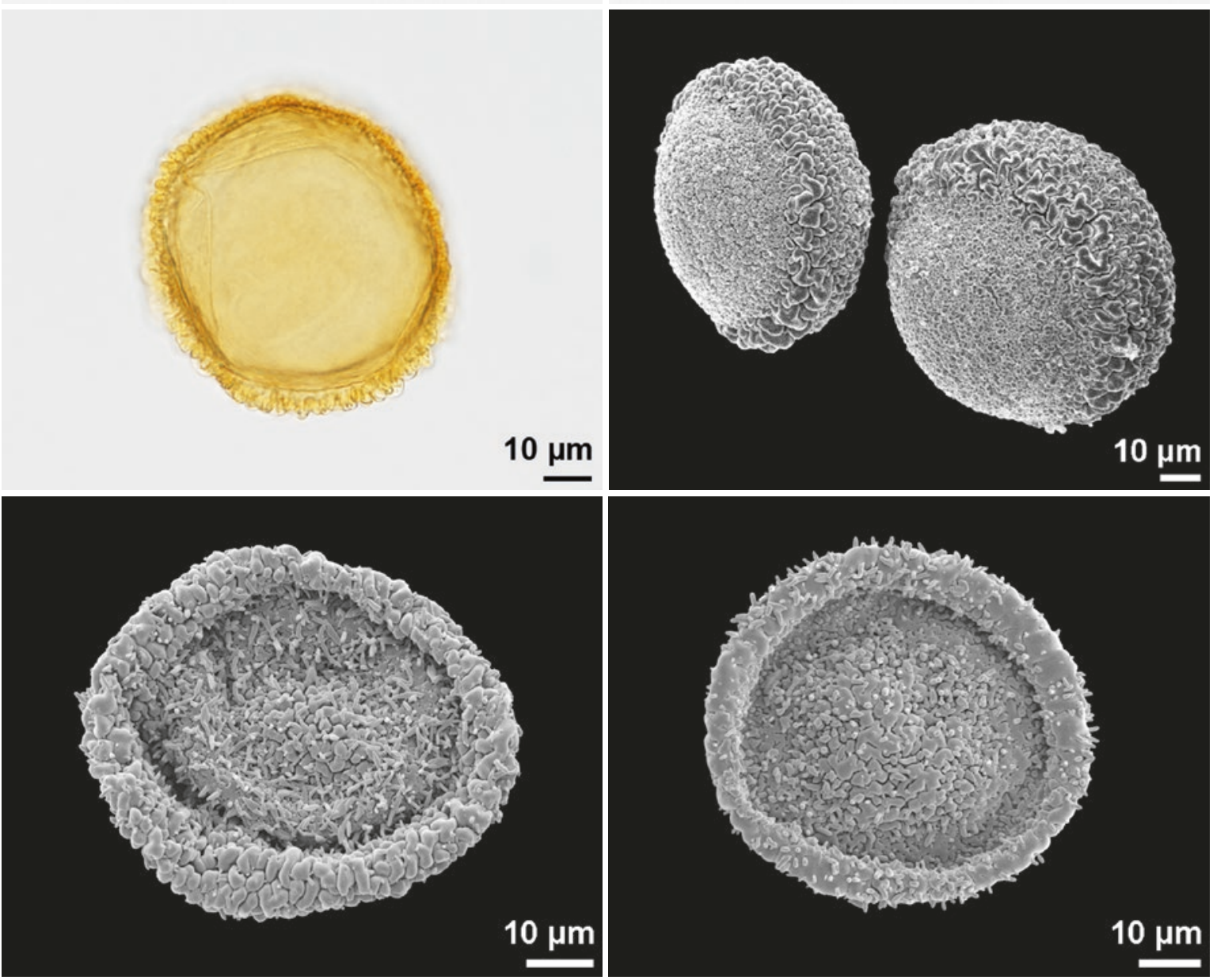

Tsuga sp., Pinaceae

fossil, middle Miocene, Austria, polar view

- Tsuga sp., Pinaceae

fossil, middle Miocene, Austria, equatorial view

- Tsuga canadensis, Pinaceae

polar view

- Tsuga canadensis, Pinaceae

equatorial view

Tsuga sp., Pinaceae

- fossil, Miocene, China, polar view

Tsuga sp., Pinaceae

fossil, Miocene, China, polar view 
saccus, bisaccate

bisaccate: pollen grain with two sacci

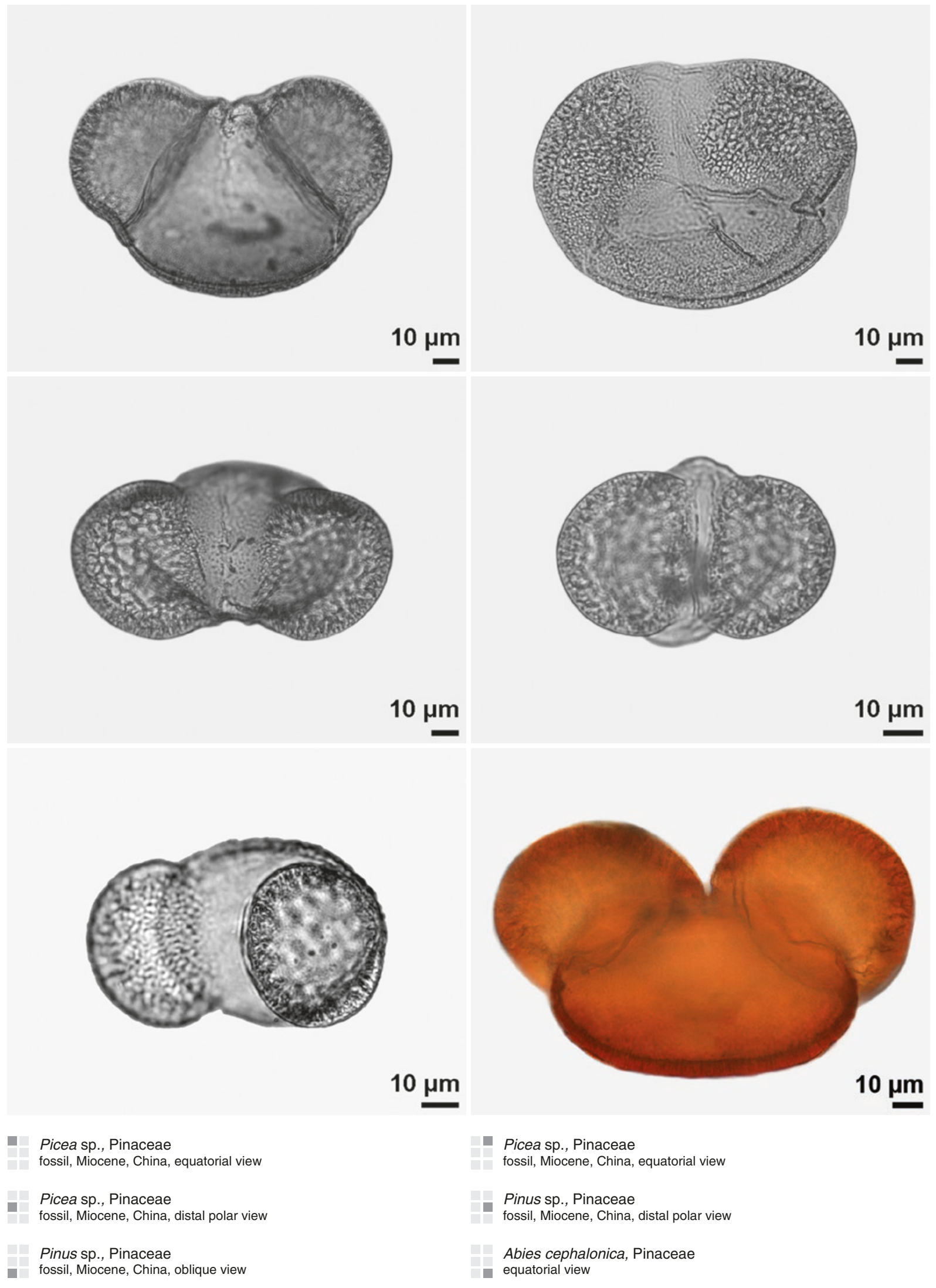



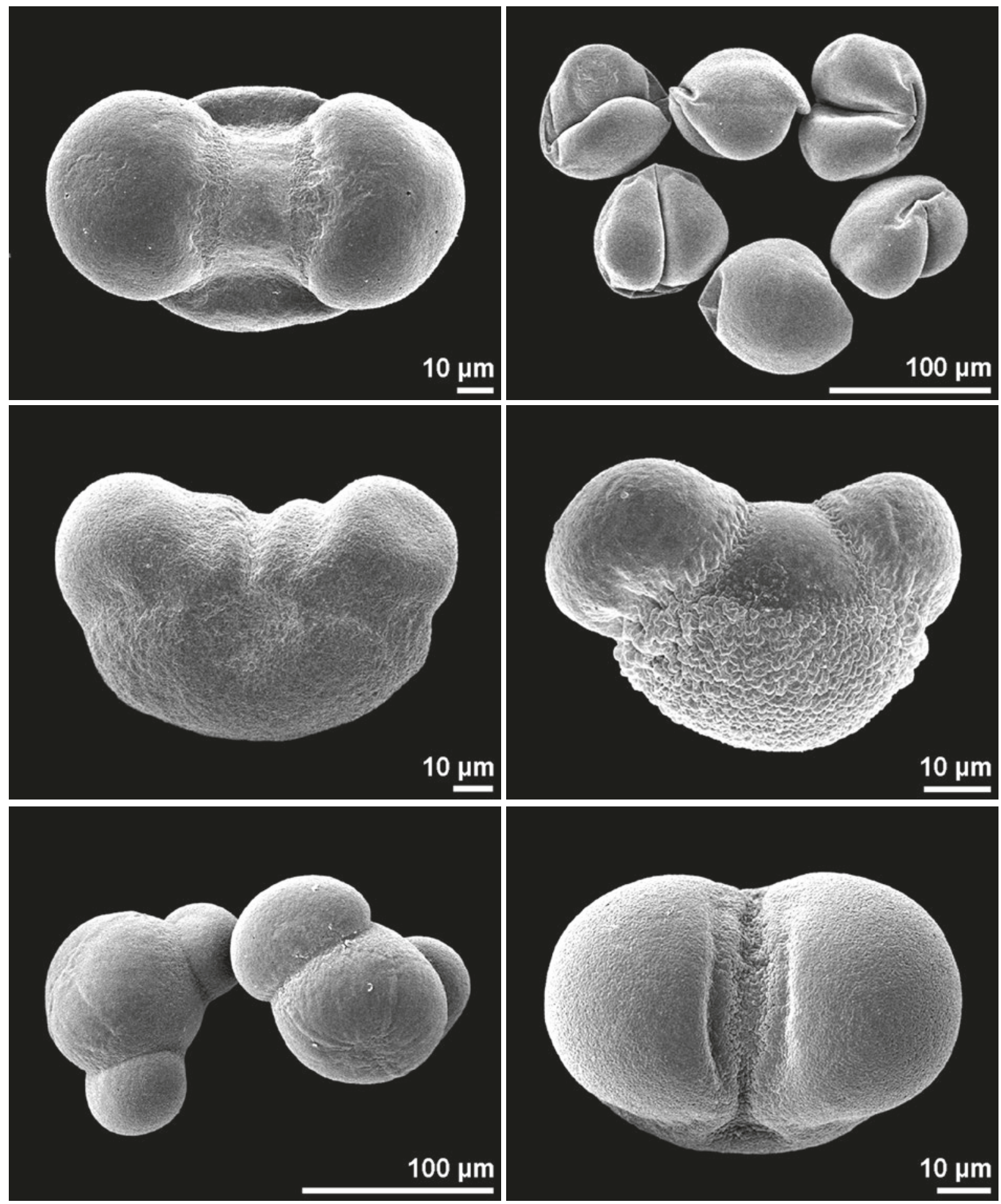

- Abies cephalonica, Pinaceae

- Picea abies, Pinaceae distal polar view

dry pollen

- Pinus mugo, Pinaceae

Picea abies, Pinaceae equatorial view

equatorial view

Picea pungens, Pinaceae

- distal polar view 

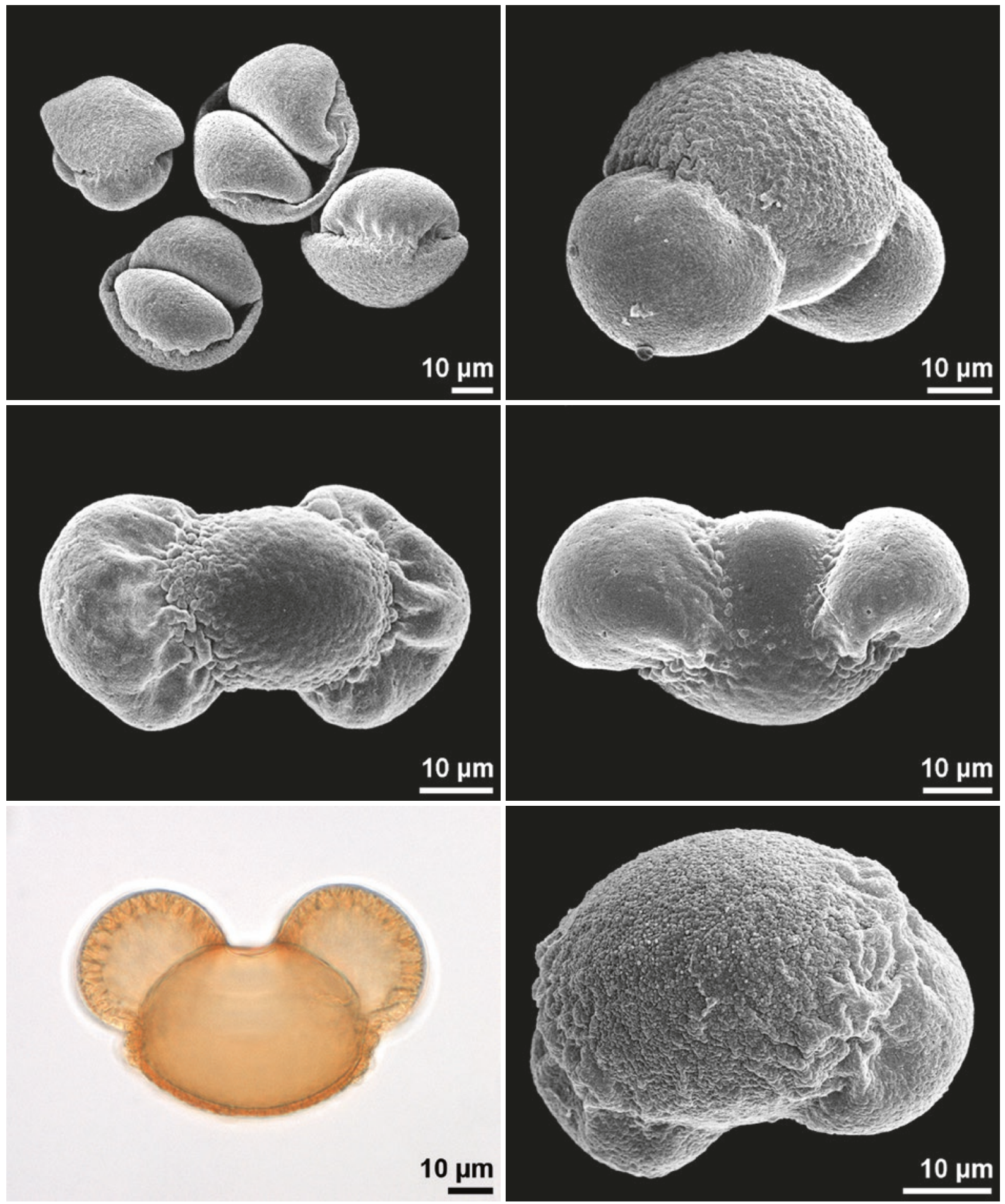

- Pinus heldreichii, Pinaceae

- Pinus nigra, Pinaceae

dry pollen

equatorial view

Podocarpus sp., Podocarpaceae

proximal polar view

- Podocarpus sp., Podocarpaceae

equatorial view

Pinus mugo, Pinaceae

equatorial view

Pinus contorta, Pinaceae

oblique proximal polar view 


\section{saccus, trisaccate}

trisaccate: pollen grain with three sacci
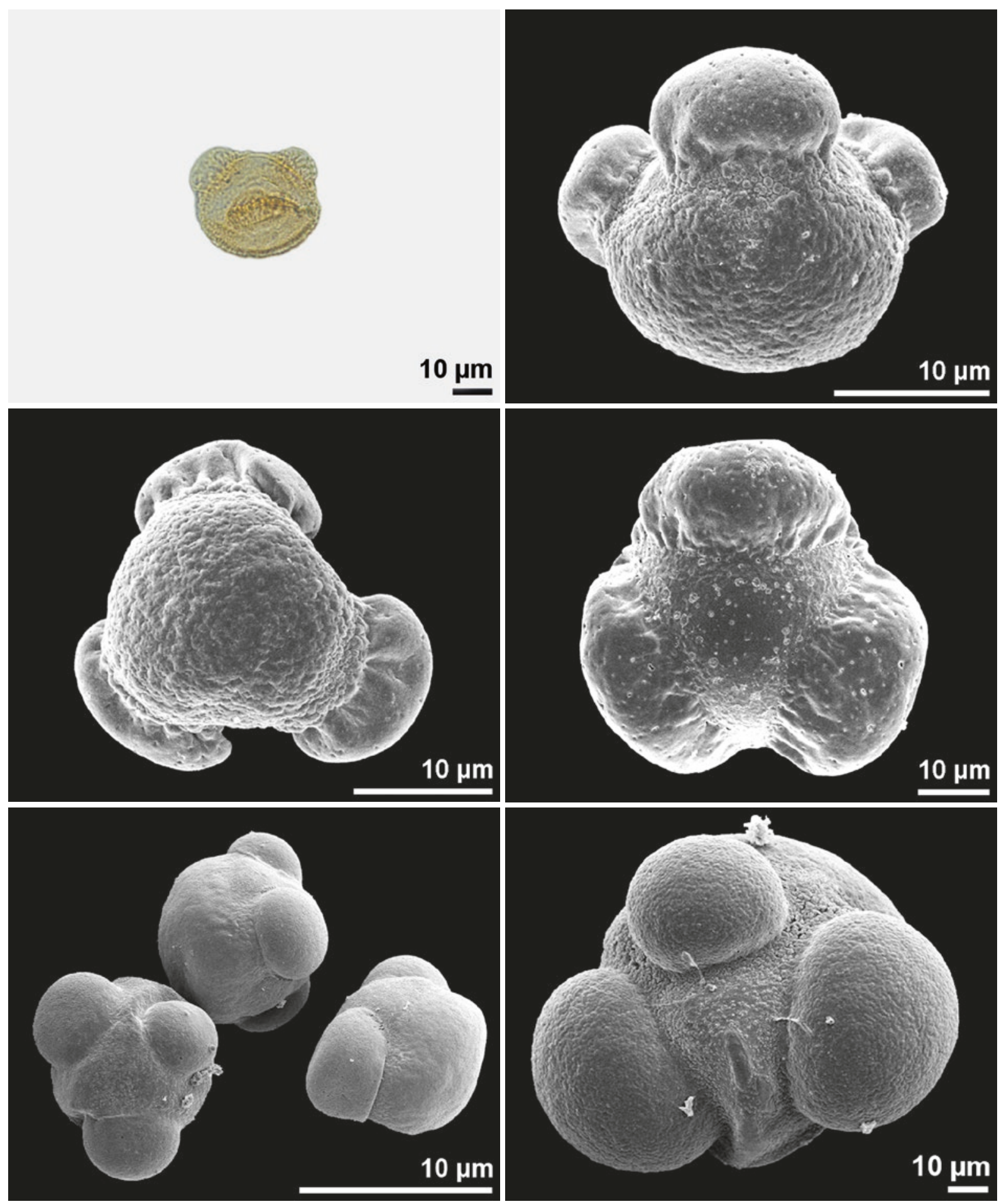

- Podocarpaceae

fossil, early Miocene, South-Africa, oblique view

- Pherosphaera hookeriana, Podocarpaceae equatorial view

Dacrycarpus dacrydioides, Podocarpaceae

Pherosphaera hookeriana, Podocarpaceae proximal polar view

distal polar view

Abies concolor, Pinaceae

Abies concolor, Pinaceae

distal polar view 
infoldings, apertures sunken

infoldings (dry pollen): consequence of harmomegathy in dry condition
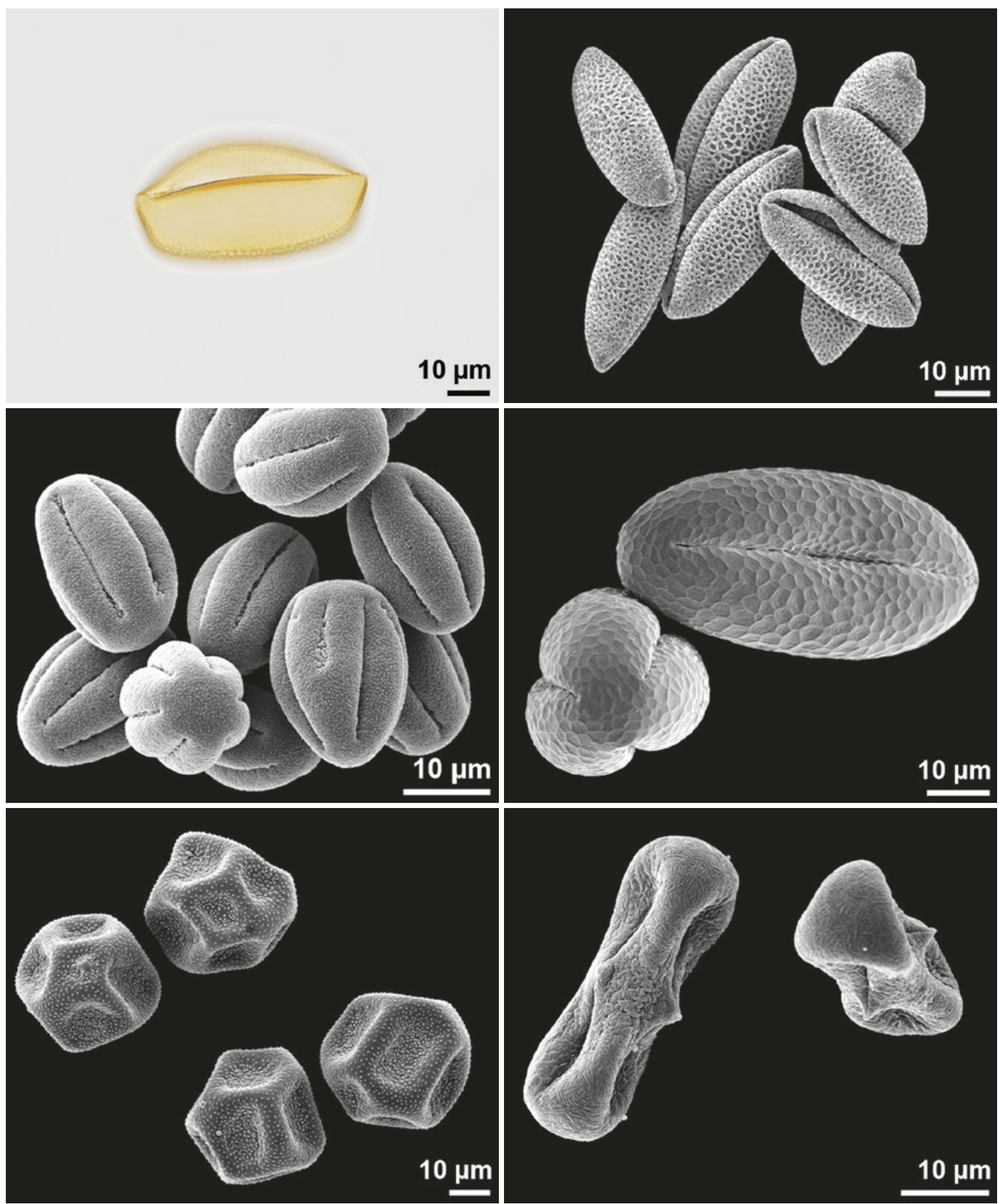

\footnotetext{
- Fritillaria pontica, Liliaceae sulcate

- Galium odoratum, Rubiaceae colpate

Roemeria hybrida, Papaveraceae - pantoporate
}

\footnotetext{
- Veratrum album, Melanthiaceae sulcate Sparmannia africana, Tiliaceae
colporate

Bifora radians, Apiaceae

- tricolporate
} 

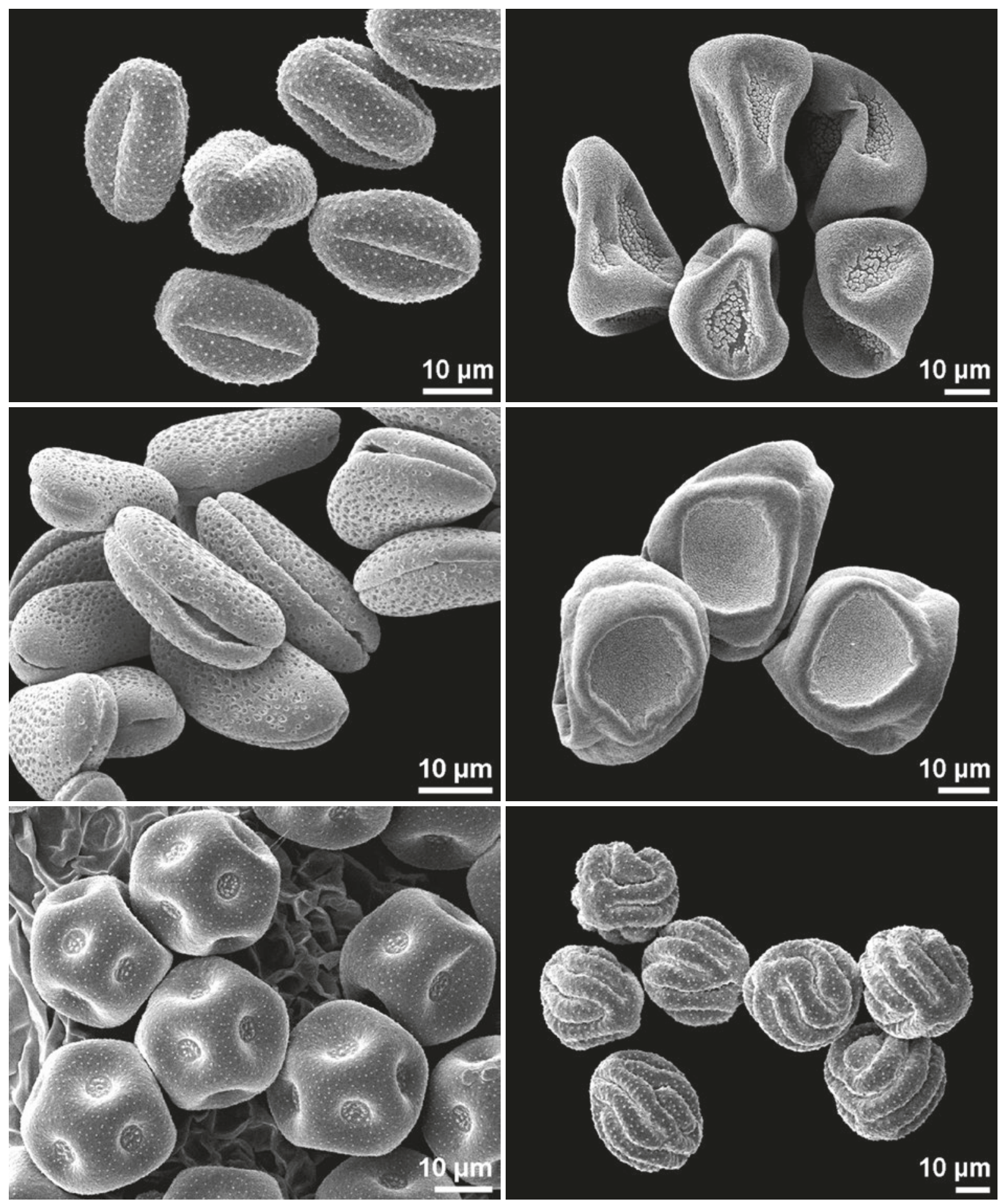

- Artemisia pontica, Asteraceae

- Carex alba, Cyperaceae colporate pseudomonads, poroidate

Luzula sylvatica, Juncaceae
tetrads, ulcerate

Lachenalia aloides, Asparagaceae sulcate

Moehringia muscosa, Caryophyllaceae

Anemone hortensis, Ranunculaceae

pantoporate

spiraperturate 
infoldings, boat-shaped
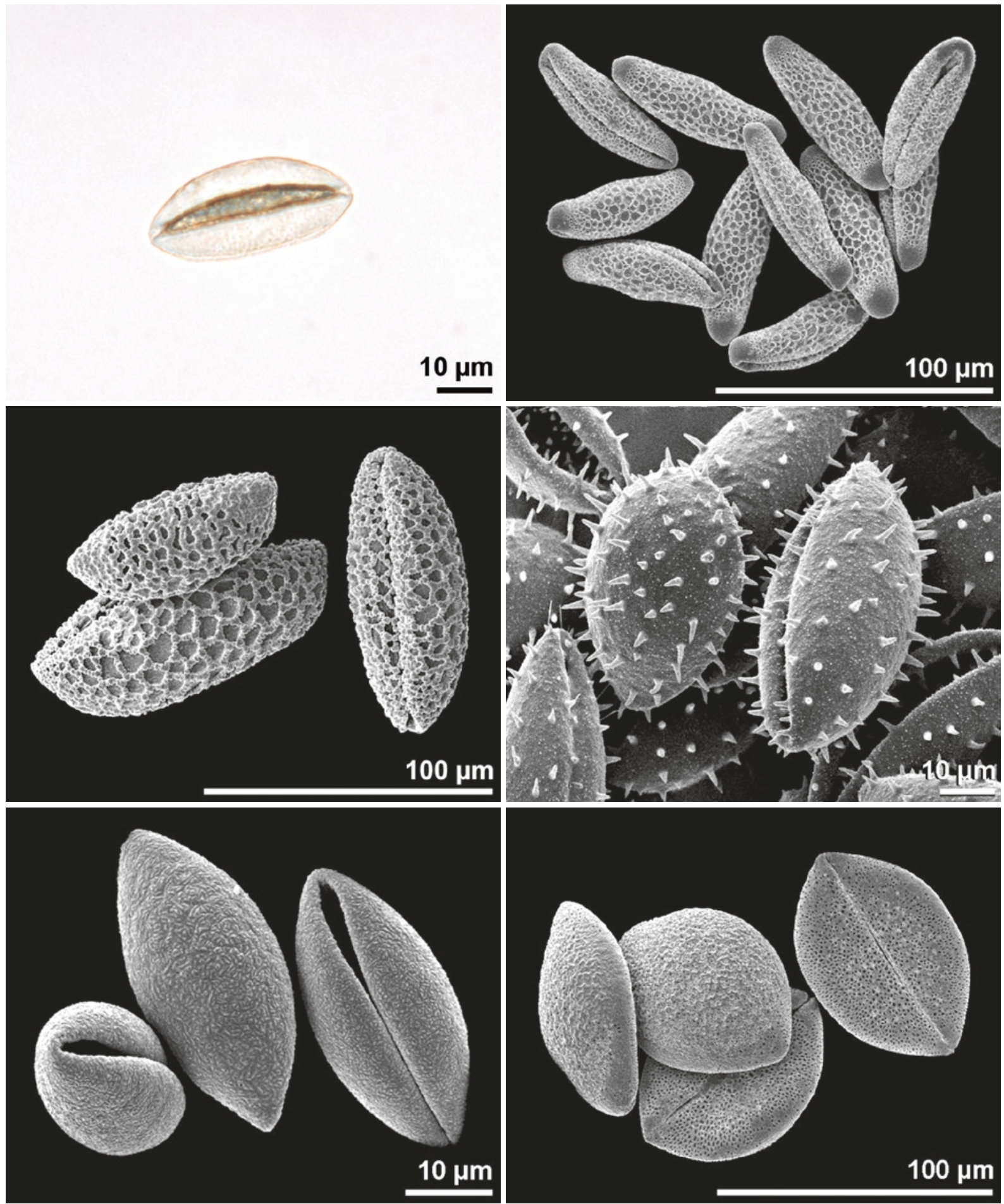

- Dracontium asperum, Araceae

- Billbergia seidelii, Bromeliaceae

sulcate

sulcate

Lilium candidum, Liliaceae
sulcate

nuphar lutea, Nymphaeaceae

Ginkgo biloba, Ginkgoaceae

sulcate

sulcate

Asphodeline lutea, Xanthorrhoeaceae

sulcate 

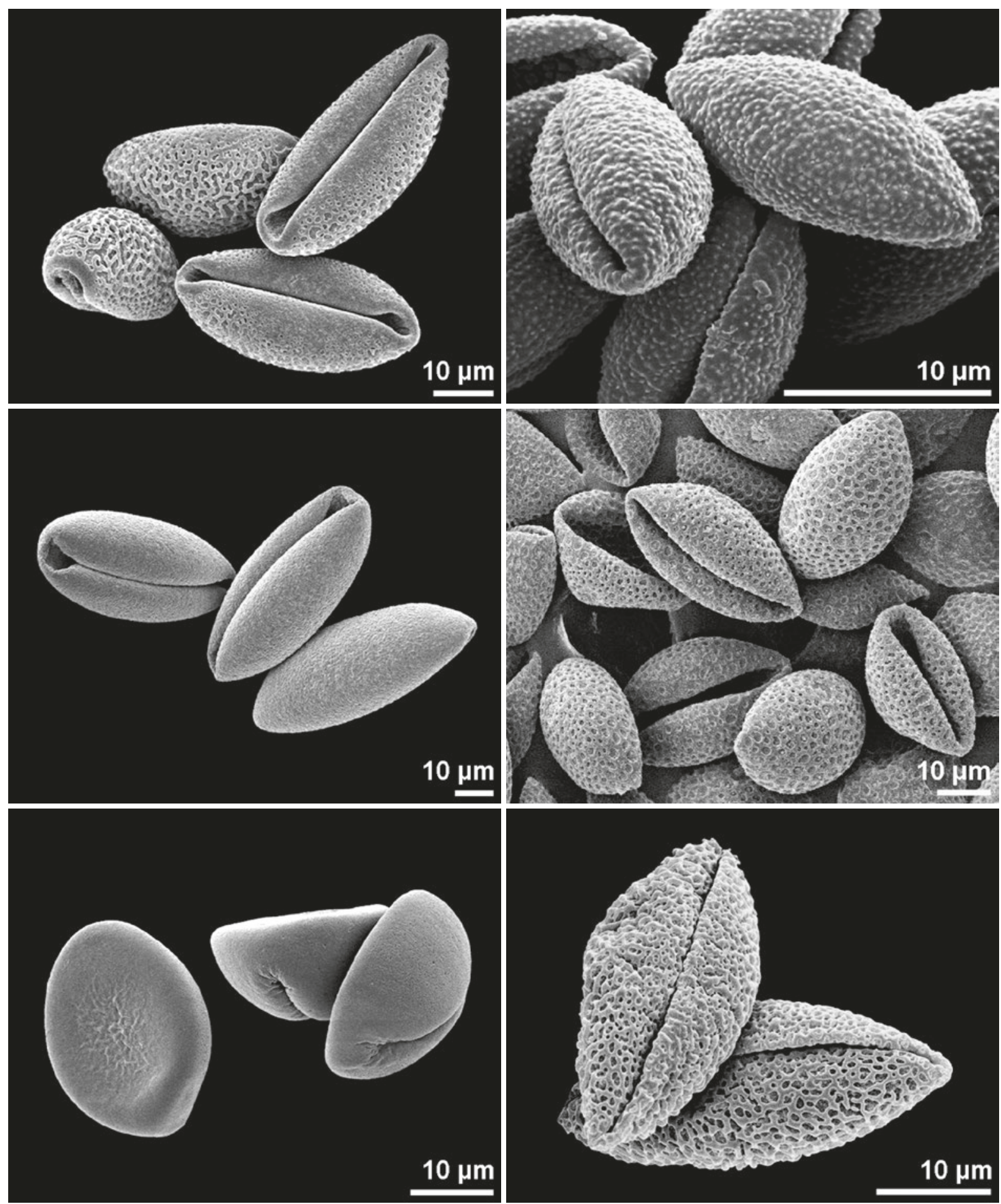

- Lysichiton americanus, Araceae

- Piper nigrum, Piperaceae sulcate sulcate Sparganium erectum, Typhaceae
ulcerate

Symplocarpus foetidus, Araceae - sulcate 

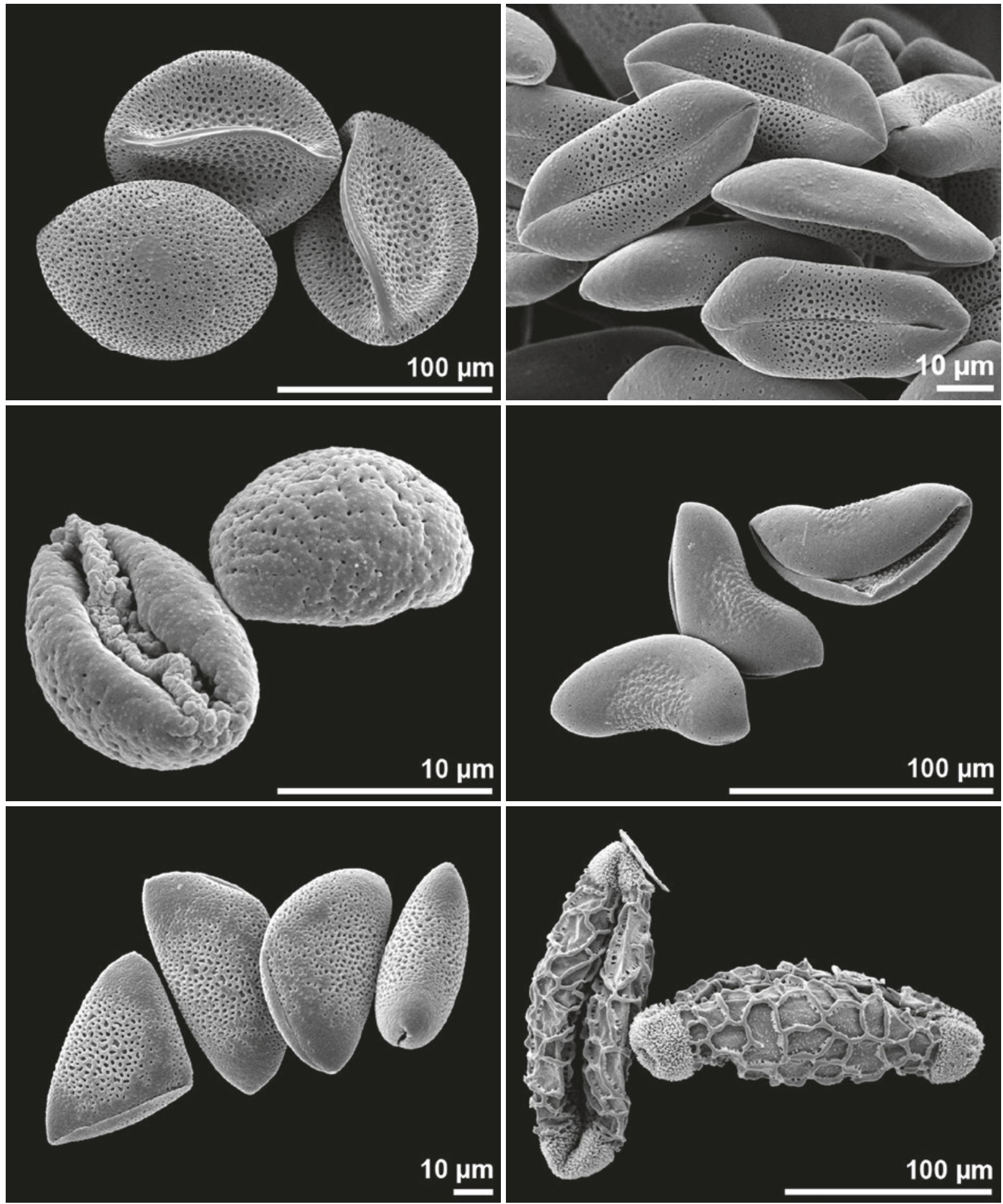

- Asphodelus fistulosus, Xanthorrhoeaceae sulcate

Eremurus thiodanthus, Xanthorrhoeaceae sulcate

Wachendorfia thyrsiflora, Haemodoraceae

Piper auritum, Piperaceae sulcate

sulcate

Hymenocallis tubiflora, Amaryllidaceae

sulcate 
infoldings, cup-shaped
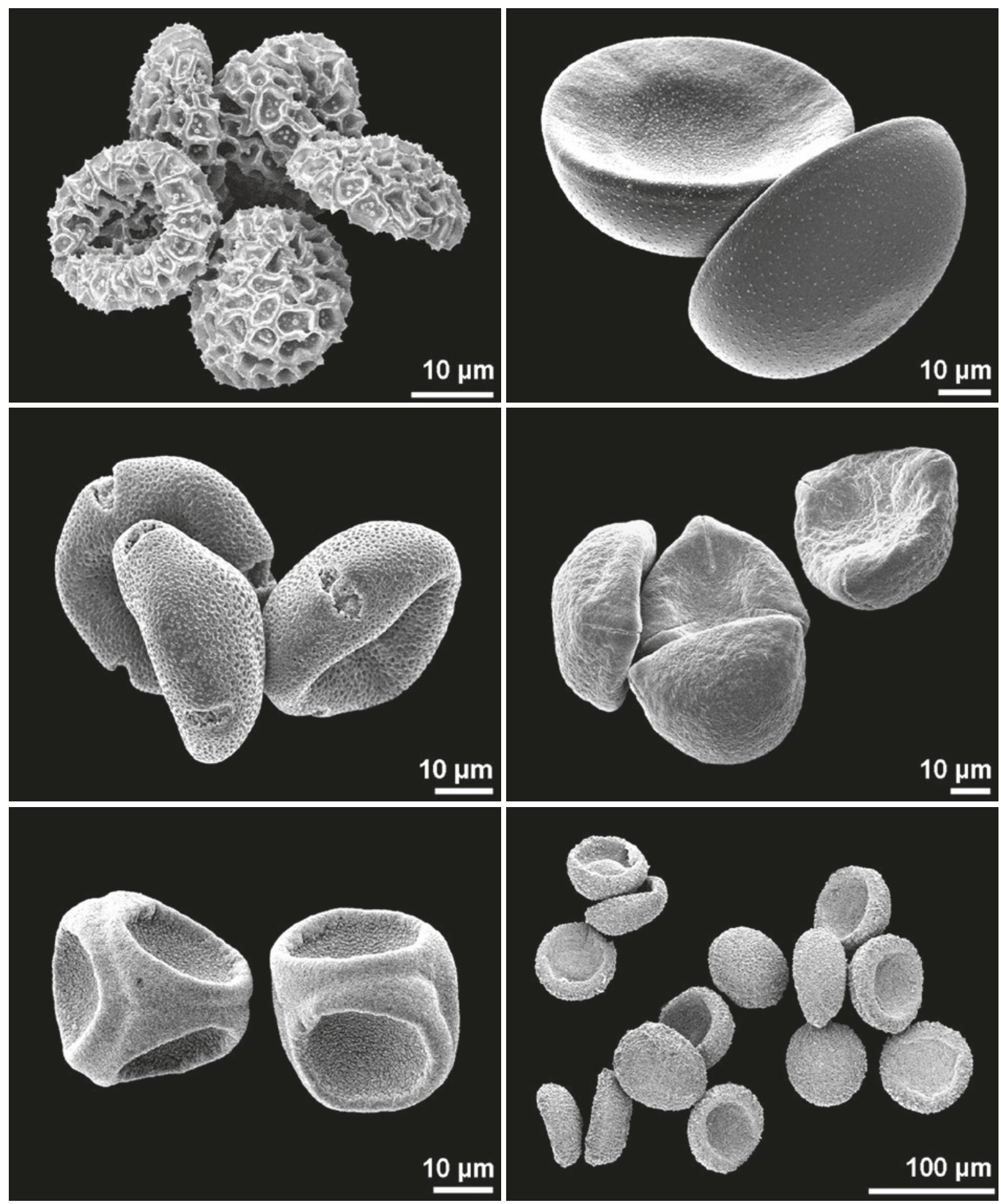

- Bougainvillea sp., Nyctaginaceae

colpate

Heliconia sp., Heliconiaceae

ulcerate

Tilia euchlora, Malvaceae colporate

Elaeagnus angustifolia, Elaeagnaceae colporate

Tsuga canadensis, Pinaceae

- leptoma 

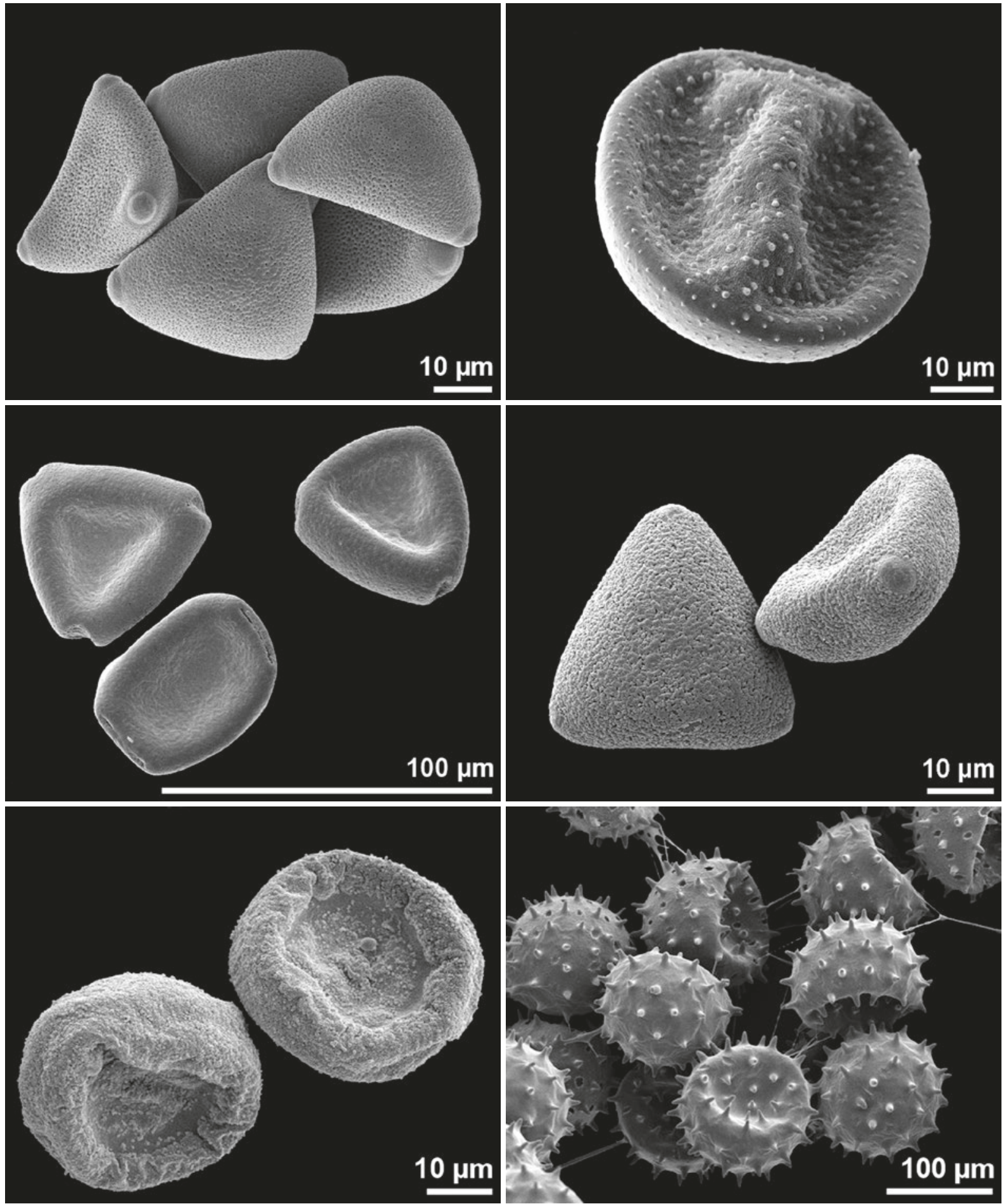

- Adenanthos sericeus, Proteaceae

- Heliconia stricta, Heliconiaceae porate

ulcerate

- Petrea volubilis, Verbenaceae brevicolpate

Leucadendron brunioides, Proteaceae porate

Cunninghamia lanceolata, Cupressaceae - leptoma

Hibiscus schizopetalus, Malvaceae

porate 
infoldings, interapertural area sunken
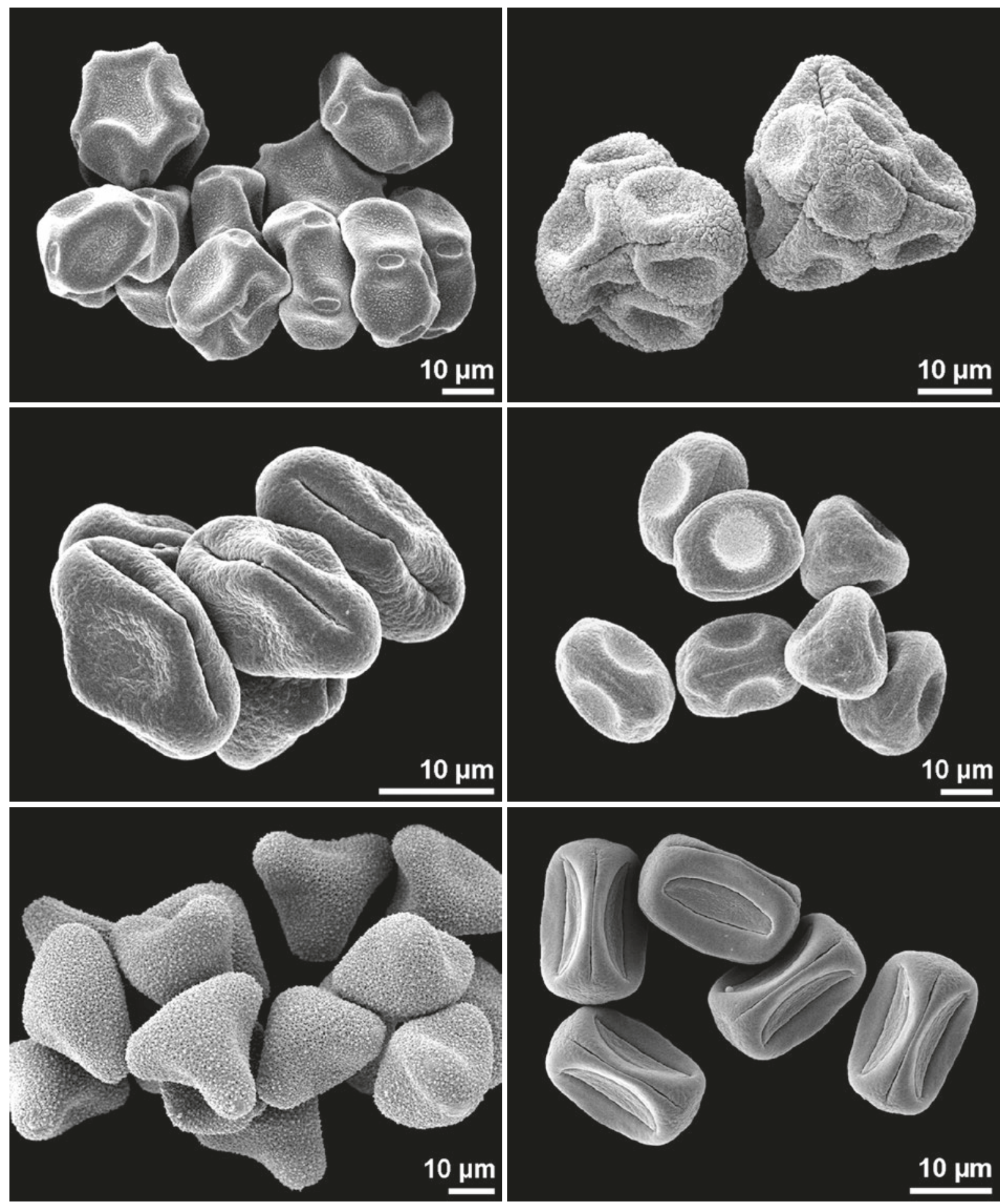

- Alnus glutinosa, Betulaceae

Erica arborea, Ericaceae

porate

tetrads, colporate

Bupleurum rotundifolium, Apiaceae
colporate

Melampyrum arvense, Orobanchaceae
colpate

Leucadendron discolor, Proteaceae

norate

Melastoma sanguineum, Melastomataceae

colporate, heteroaperturate 

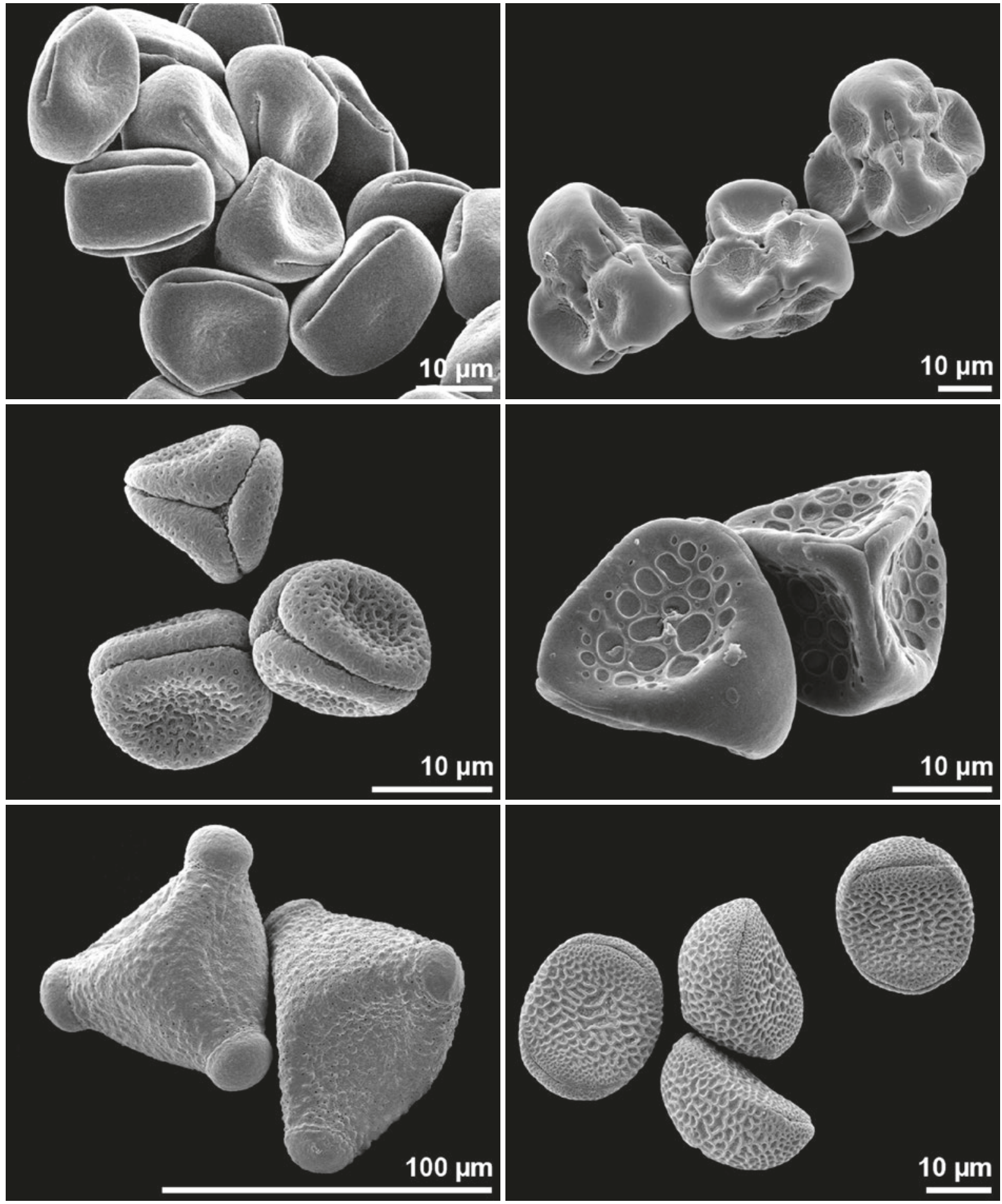

- Verbena officinalis, Verbenaceae

- Tsusiophyllum tanakae, Ericaceae colporate tetrads, colporate

- Thesium arvense, Santalaceae

Ardisia crenata, Primulaceae syncolporate

colpate, triradiate colpus

Grevillea banksii, Proteaceae

porate

Tropaeolum moritzianum, Tropaeolaceae

colpate 
infoldings, irregular
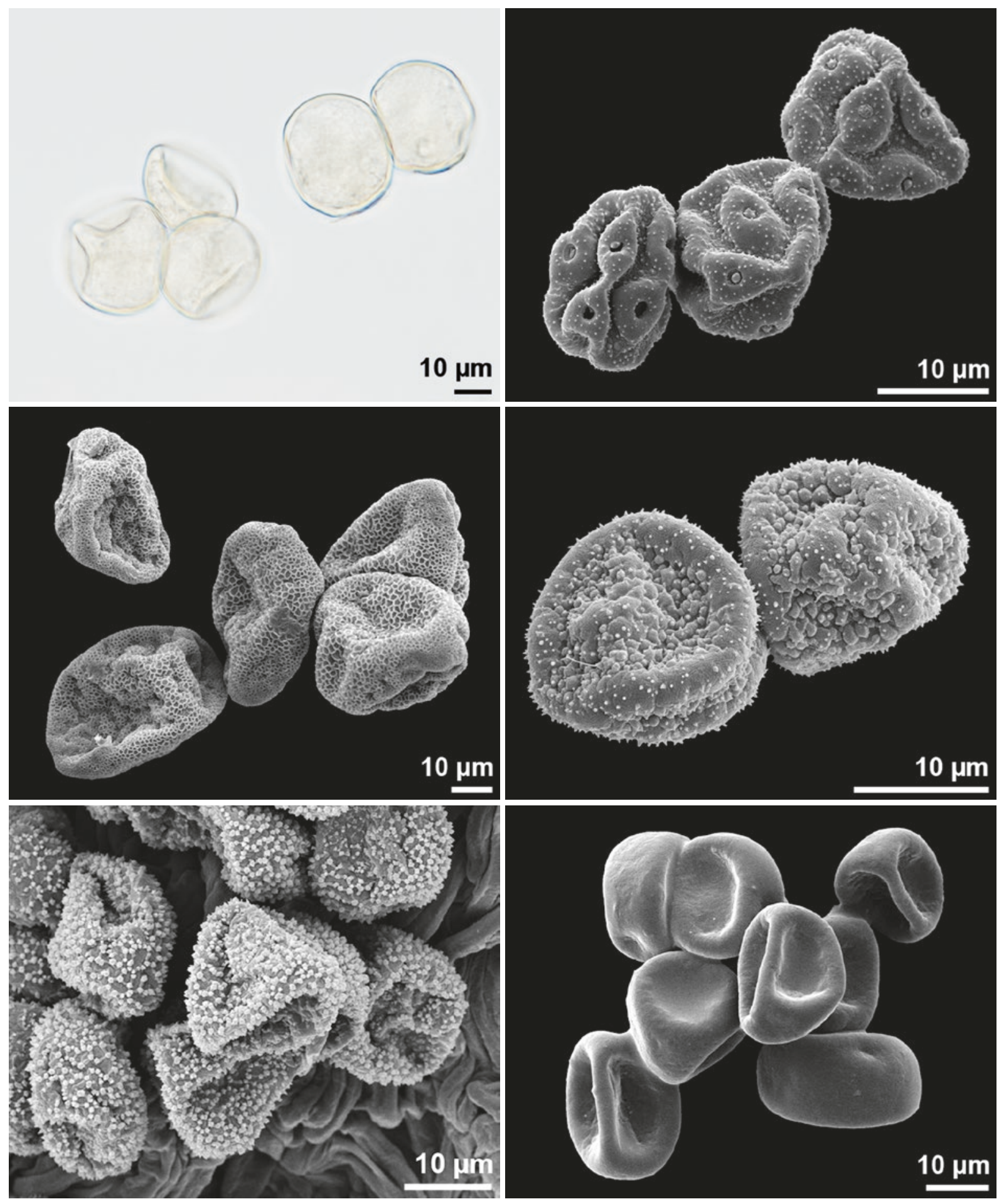

- Poaceae

Dorstenia contrajerva, Moraceae pantoporate

Guzmania elvallensis, Bromeliaceae

Smilax spinosa, Smilacaceae aperture condition unclear

Callitriche stagnalis, Plantaginaceae inaperturate

Vanilla planifolia, Orchidaceae - porate 

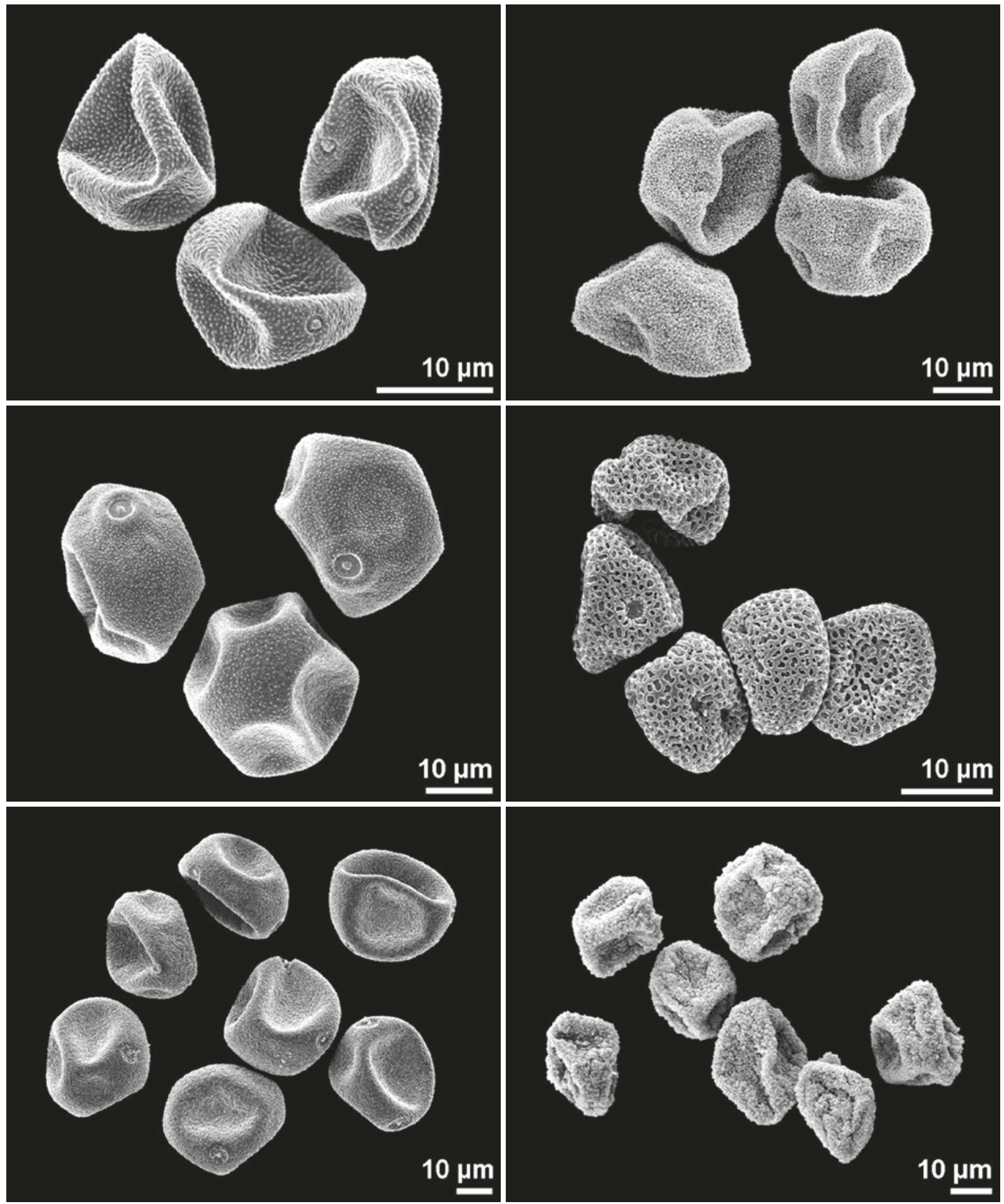

- Urtica dioica, Urticaceae

- Populus alba, Salicaceae porate inaperturate

Sesleria albicans, Poaceae
ulcerate

Anthurium radicans, Araceae

Orobanche hederae, Orobanchaceae

- inaperturate

- porate 
Open Access This chapter is licensed under the terms of the Creative Commons Attribution 4.0 International License (http://creativecommons.org/licenses/by/4.0/), which permits use, sharing, adaptation, distribution and reproduction in any medium or format, as long as you give appropriate credit to the original author(s) and the source, provide a link to the Creative Commons license and indicate if changes were made.

The images or other third party material in this chapter are included in the chapter's Creative Commons license, unless indicated otherwise in a credit line to the material. If material is not included in the chapter's Creative Commons license and your intended use is not permitted by statutory regulation or exceeds the permitted use, you will need to obtain permission directly from the copyright holder.

(c) (i) 